\title{
No Agent Left Behind: Dynamic Fair Division of Multiple Resources
}

\section{Ian Kash}

Microsoft Research Cambridge, UK

Ariel D. Procaccia

Carnegie Mellon University, USA

Nisarg Shah

Carnegie Mellon University, USA
IANKASH@MICROSOFT.COM

ARIELPRO@CS.CMU.EDU

NKSHAH@CS.CMU.EDU

\begin{abstract}
Recently fair division theory has emerged as a promising approach for allocation of multiple computational resources among agents. While in reality agents are not all present in the system simultaneously, previous work has studied static settings where all relevant information is known upfront. Our goal is to better understand the dynamic setting. On the conceptual level, we develop a dynamic model of fair division, and propose desirable axiomatic properties for dynamic resource allocation mechanisms. On the technical level, we construct two novel mechanisms that provably satisfy some of these properties, and analyze their performance using real data. We believe that our work informs the design of superior multiagent systems, and at the same time expands the scope of fair division theory by initiating the study of dynamic and fair resource allocation mechanisms.
\end{abstract}

\section{Introduction}

The question of how to fairly divide goods or resources has been the subject of intellectual curiosity for millennia. While early solutions can be traced back to ancient writings, rigorous approaches to fairness were proposed only as late as the mid Twentieth Century, by mathematicians and social scientists. Over time, fair division has emerged as an influential subfield of microeconomic theory. In the last few years fair division has also attracted the attention of AI researchers (see, e.g., Chevaleyre, Endriss, Estivie, \& Maudet, 2007; Procaccia, 2009; Chen, Lai, Parkes, \& Procaccia, 2010; Moulin, 2003; Brams \& Taylor, 1996), who envision applications of fair division in multiagent systems (Chevaleyre, Dunne, Endriss, Lang, Lemaître, Maudet, Padget, Phelps, Rodríguez-Aguilar, \& Sousa, 2006). However, fair division theory has seen relatively few applications to date.

It is only very recently that an exciting combination of technological advances and theoretical innovations has pointed the way towards concrete applications of fair division. In modern data centers, clusters, and grids, multiple computational resources (such as CPU, memory, and network bandwidth) must be allocated among heterogeneous agents. Agents' demands for resources are typically highly structured, as we explain below. Several recent papers (Gutman \& Nisan, 2012; Ghodsi, Zaharia, Hindman, Konwinski, Shenker, \& Stoica, 2011; Parkes, Procaccia, \& Shah, 2014; Dolev, Feitelson, Halpern, Kupferman, \& Linial, 2012) suggest that classic fair division mechanisms possess excellent properties in these environments, in terms of their fairness guarantees as well as their game-theoretic properties.

Nevertheless, some aspects of realistic computing systems are beyond the current scope of fair division theory. Perhaps most importantly, the literature does not capture the dynamics of these systems. Indeed, it is typically not the case that all the agents are present in the system at any 
given time; agents may arrive and depart, and the system must be able to adjust the allocation of resources. Even on the conceptual level, dynamic settings challenge some of the premises of fair division theory. For example, if one agent arrives before another, the first agent should intuitively have priority; what does fairness mean in this context? We introduce the concepts that are necessary to answer this question, and design novel mechanisms that satisfy our proposed desiderata. Our contribution is therefore twofold: we design more realistic resource allocation mechanisms for multiagent systems that provide theoretical guarantees, and at the same time we expand the scope of fair division theory to capture dynamic settings.

\subsection{Overview of Model and Results}

As in previous papers (e.g., Ghodsi et al., 2011; Parkes et al., 2014), we assume that agents demand the resources in fixed proportions. Such Leontief preferences - as they are known in economics are easily justified in typical settings where agents must run many instances of a single task (e.g., map jobs in the MapReduce framework). Hence, for example, an agent that requires twice as much CPU as RAM to run a task prefers to be allocated 4 CPU units and 2 RAM units to 2 CPU units and 1 RAM unit, but is indifferent between the former allocation and 5 CPU units and 2 RAM units.

We consider environments where agents arrive over time (but do not depart - see Section 7 for additional discussion of this point). We aim to design resource allocation mechanisms that make irrevocable allocations, i.e., the mechanism can allocate more resources to an agent over time, but can never take resources back.

We adapt prominent notions of fairness, efficiency, and truthfulness to our dynamic settings. For fairness, we ask for envy freeness $(E F)$, in the sense that agents like their own allocation best; and sharing incentives (SI), so that agents prefer their allocation to their proportional share of the resources. We also seek strategyproof $(S P)$ mechanisms: agents cannot gain from misreporting their demands. Finally, we introduce the notion of dynamic Pareto optimality (DPO): if $k$ agents are entitled to $k / n$ of each resource, the allocation should not be dominated (in a sense that will be formalized later) by allocations that divide these entitlements. Our first result (in Section 3) is an impossibility: DPO and EF are incompatible. We proceed by relaxing each of these properties.

In Section 4, we relax the EF property. The new dynamic property, which we call dynamic EF $(D E F)$, allows an agent to envy another agent that arrived earlier, as long as the former agent was not allocated resources after the latter agent's arrival. We construct a new mechanism, DYNAMIC DRF, and prove that it satisfies SI, DEF, SP, and DPO.

In Section 5, we relax the DPO property. Our cautious DPO (CDPO) notion allows allocations to only compete with allocations that can ultimately guarantee EF, regardless of the demands of future agents. We design a mechanism called CAUTIOUS LP, and show that it satisfies SI, EF, SP, and CDPO. In a sense, our theoretical results are tight: EF and DPO are incompatible, but relaxing only one of these two properties is sufficient to enable mechanisms that satisfy both, in conjunction with SI and SP.

Despite the assumptions imposed by our theoretical model, we believe that our new mechanisms are compelling, useful guides for the design of practical resource allocation mechanisms in realistic settings. Indeed, in Section 6, we test our mechanisms on real data obtained from a trace of workloads on a Google cluster, and obtain encouraging results. 


\subsection{Related Work}

Walsh (2011) proposed the problem of fair cake cutting where agents arrive, take a piece of cake, and immediately depart. The cake cutting setting deals with the allocation of a single, heterogeneous divisible resource; contrast with our setting, which deals with multiple, homogeneous divisible resources. Walsh suggested several desirable properties for cake cutting mechanisms in this setting, and showed that adaptations of classic mechanisms achieve these properties (Walsh also pointed out that allocating the whole cake to the first agent achieves the same properties). In particular, his notion of forward envy freeness, which is discussed below, is related to our notion of dynamic envy freeness.

The networking community has studied the problem of fairly allocating a single homogeneous resource in a queuing model where each agent's task requires a given number of time units to be processed. In other words, in these models tasks are processed over time, but demands stay fixed, and there are no other dynamics such as agent arrivals and departures. The well-known fair queuing solution (Demers, Keshav, \& Shenker, 1989) allocates one unit per agent in successive round-robin fashion. This solution has also been analyzed by economists (Moulin \& Stong, 2002).

Previous papers on the allocation of multiple resources study a static setting. For example, Ghodsi et al. (2011) proposed the dominant resource fairness (DRF) mechanism, which guarantees a number of desirable theoretical properties. Li and Xue (2013) presented characterizations of mechanisms satisfying various desiderata while Wong et al. (2012) analyzed the classic tradeoff between fairness and efficiency, both in generic frameworks that capture DRF as a special case. Parkes et al. (2014) extended DRF in several ways, and in particular studied the case of indivisible tasks. Finally, DRF has also been extended to the queuing domain (Ghodsi, Sekar, Zaharia, \& Stoica, 2012) and to incorporate job placement considerations (Ghodsi, Zaharia, Shenker, \& Stoica, 2013), but these generalizations also use a static setting. Recently, Zahedi and Lee (2014) applied the concept of Competitive Equilibrium from Equal Outcomes (CEEI) in the case of Cobb-Douglas utilities to achieve properties similar to DRF. They empirically show that these utilities are well suited for modeling user preferences over hardware resources such as cache capacity and memory bandwidth. Dolev et al. (2012) defined a notion of fairness that is different from the one considered in DRF. They also proved that a fair allocation according to this new notion is always guaranteed to exist in a static setting. Gutman and Nisan (2012) gave a polynomial time algorithm to find such an allocation, and also considered generalizations of DRF in a more general model of utilities. We elaborate on several of these results below.

\section{Preliminaries}

In our setting, each agent has a task that requires fixed amounts of different resources. The utility of the agent depends on the quantity (possibly fractional) of its tasks that it can execute given the allocated resources. Formally, denote the set of agents by $N=\{1, \ldots, n\}$, and the set of resources by $R,|R|=m$. Let $D_{i r}$ denote the ratio between the maximum amount of resource $r$ agent $i$ can use given the amounts of other resources present in the system and the total amount of that resource available in the system, either allocated or free. In other words, $D_{i r}$ is the fraction of resource $r$ required by agent $i$. Following Ghodsi et al. (2011), the dominant resource of agent $i$ is defined as the resource $r$ that maximizes $D_{i r}$, and the fraction of dominant resource allocated to agent $i$ is called its dominant share. Following Parkes et al. (2014), the (normalized) demand vector of agent $i$ is given by $\mathbf{d}_{i}=\left\langle d_{i 1}, \ldots, d_{i m}\right\rangle$, where $d_{i r}=D_{i r} /\left(\max _{r^{\prime}} D_{i r^{\prime}}\right)$ for each resource $r$. Let $\mathcal{D}$ be the 
set of all possible normalized demand vectors. Let $\mathbf{d}_{\leq k}=\left\langle\mathbf{d}_{1}, \ldots, \mathbf{d}_{k}\right\rangle$ denote the demand vectors of agents 1 through $k$. Similarly, let $\mathbf{d}_{>k}=\left\langle\mathbf{d}_{k+1}, \ldots, \mathbf{d}_{n}\right\rangle$ denote the demand vectors of agents $k+1$ through $n$.

An allocation A allocates a fraction $A_{i r}$ of resource $r$ to agent $i$, subject to the feasibility condition $\sum_{i \in N} A_{i r} \leq 1$ for all $r \in R$. Throughout the paper we assume that resources are divisible and that each agent requires a positive amount of each resource, i.e., $d_{i r}>0$ for all $i \in N$ and $r \in R$. Under such allocations, our model for preferences coincides with the domain of Leontief preferences, where the utility of an agent for its allocation vector $\mathbf{A}_{i}$ is given by

$$
u_{i}\left(\mathbf{A}_{i}\right)=\max \left\{y \in \mathbb{R}_{+}: \forall r \in R, A_{i r} \geq y \cdot d_{i r}\right\}
$$

In words, the utility of an agent is the fraction of its dominant resource that it can actually use, given its proportional demands and its allocation of the various resources. However, we do not rely on an interpersonal comparison of utilities; an agent's utility function simply induces ordinal preferences over allocations, and its exact value is irrelevant.

We say that an allocation $\mathbf{A}$ is Pareto-dominated by another allocation $\mathbf{A}^{\prime}$ if $u_{i}\left(\mathbf{A}_{i}^{\prime}\right) \geq u_{i}\left(\mathbf{A}_{i}\right)$ for every agent $i$, and $u_{j}\left(\mathbf{A}_{j}^{\prime}\right)>u_{j}\left(\mathbf{A}_{j}\right)$ for some agent $j$. For allocations $\mathbf{A}$ over agents in $S \subseteq N$ and $\mathbf{A}^{\prime}$ over agents in $T \subseteq N$ such that $S \subseteq T$, we say that $\mathbf{A}^{\prime}$ is an extension of $\mathbf{A}$ to $T$ if $A_{i r}^{\prime} \geq A_{i r}$ for every agent $i \in S$ and every resource $r$. When $S=T$, we simply say that $\mathbf{A}^{\prime}$ is an extension of $\mathbf{A}$. An allocation $\mathbf{A}$ is called non-wasteful if for every agent $i$ there exists $y \in \mathbb{R}_{+}$such that for all $r \in R, A_{i r}=y \cdot d_{i r}$. For a non-wasteful allocation, the utility of an agent is the share of its dominant resource allocated to the agent. Also, if $\mathbf{A}$ is a non-wasteful allocation then for all $i \in N$,

$$
u_{i}\left(\mathbf{A}_{i}^{\prime}\right)>u_{i}\left(\mathbf{A}_{i}\right) \Rightarrow \forall r \in R, A_{i r}^{\prime}>A_{i r}
$$

\section{Dynamic Resource Allocation: A New Model}

We consider a dynamic resource allocation model where agents arrive at different times and do not depart (see Section 7 for a discussion of this point). We assume that agent 1 arrives first, then agent 2 , and in general agent $k$ arrives after agents $1, \ldots, k-1$; we say that agent $k$ arrives in step $k$. An agent reports its demand when it arrives and the demand does not change over time. Thus, at step $k$, demand vectors $\mathbf{d}_{\leq k}$ are known, and demand vectors $\mathbf{d}_{>k}$ are unknown. A dynamic resource allocation mechanism operates as follows. At each step $k$, the mechanism takes as input the reported demand vectors $\mathbf{d}_{\leq k}$ and outputs an allocation $\mathbf{A}^{k}$ over the agents present in the system. Crucially, we assume that allocations are irrevocable, i.e., $A_{i r}^{k} \geq A_{i r}^{k-1}$ for every step $k \geq 2$, every agent $i \leq k-1$, and every resource $r$. We also assume that the mechanism knows the total number of agents $n$ in advance.

Irrevocability can be justified in various settings, e.g., in cases where resources are committed to long-term projects. One example is that of a research cluster shared between faculty members at a university. In such a cluster, the total number of faculty members who can access the cluster (denoted $n$ in our setting) is known to the mechanism in advance - as we assume in our model. Another important setting where irrevocability becomes a necessary assumption is the case of divisible consumable resources. In this case, an agent may consume the resources it receives in a certain step, so they cannot be reclaimed later on. 
Previous work on static resource allocation (e.g., Ghodsi et al., 2011; Parkes et al., 2014) focused on designing mechanisms that satisfy four prominent desiderata. Three of these - two fairness properties and one game-theoretic property — immediately extend to the dynamic setting.

1. Sharing Incentives (SI). We say that a dynamic allocation mechanism satisfies SI if $u_{i}\left(\mathbf{A}_{i}^{k}\right) \geq$ $u_{i}(\langle 1 / n, \ldots, 1 / n\rangle)$ for all steps $k$ and all agents $i \leq k$. In words, when an agent arrives it receives an allocation that it likes at least as much as an equal split of the resources. This models a setting where agents have made equal contributions to the system and hence have equal entitlements. In such cases, the contributions are typically recorded, which allows the mechanism to know the total number of agents $n$ in advance, as assumed in our setting.

2. Envy Freeness $(E F)$. A dynamic allocation mechanism is EF if $u_{i}\left(\mathbf{A}_{i}^{k}\right) \geq u_{i}\left(\mathbf{A}_{j}^{k}\right)$ for all steps $k$ and all agents $i, j \leq k$, that is, an agent that is present would never prefer the allocation of another agent.

3. Strategyproofness (SP). A dynamic allocation mechanism is SP if no agent can misreport its demand vector and be strictly better off at any step $k$, regardless of the reported demands of other agents. Formally, a dynamic allocation mechanism is SP if for any agent $i \in N$ and any step $k$, if $\mathbf{A}_{i}^{k}$ is the allocation to agent $i$ at step $k$ when agent $i$ reports its true demand vector and $\mathbf{B}_{i}^{k}$ is the allocation to agent $i$ at step $k$ when agent $i$ reports a different demand vector (in both cases all the other agents report their true demand vectors), then $u_{i}\left(\mathbf{A}_{i}^{k}\right) \geq u_{i}\left(\mathbf{B}_{i}^{k}\right)$. We avoid introducing additional notations that will not be required later.

In the static setting, the fourth prominent axiom, Pareto optimality $(P O)$, means that the mechanism's allocation is not Pareto dominated by any other allocation. Of course, in the dynamic setting it is unreasonable to expect the allocation in early stages to be Pareto undominated, because we need to save resources for future arrivals (recall that allocations are irrevocable). We believe though that the following definition naturally extends PO to our dynamic setting.

4. Dynamic Pareto Optimality (DPO). A dynamic allocation mechanism is DPO if at each step $k$, the allocation $\mathbf{A}^{k}$ returned by the mechanism is not Pareto dominated by any other allocation $\mathbf{B}^{k}$ that allocates up to a $(k / n)$-fraction of each resource among the $k$ agents present in the system. Put another way, at each step the allocation should not be Pareto dominated by any other allocation that only redistributes the collective entitlements of the agents present in the system among those agents.

It is straightforward to verify that a non-wasteful mechanism (a mechanism returning a nonwasteful allocation at each step) satisfies DPO if and only if the allocation returned by the mechanism at each step $k$ uses at least a $(k / n)$-fraction of at least one resource (the assumption of strictly positive demands plays a role here).

Before moving on to possibility and impossibility results, we give examples that illustrate how various combinations of the properties constrain the allocation of resources.

Example 1 (Satisfying Sharing Incentives (SI) and Dynamic Pareto Optimality (DPO)). In this paper, we only consider non-wasteful allocations. Hence, as described above, DPO is equivalent to allocating at least a $(k / n)$-fraction of at least one resource in every step $k$, when allocations are proportional. On the other hand, if a mechanism seeks to satisfy SI, it cannot allocate more than 
a $(k / n)$-fraction of any resource in step $k$. Indeed, if more than a $(k / n)$-fraction of resource $r$ is allocated at step $k$, and every agent arriving after step $k$ reports $r$ as its dominant resource, the mechanism would not have enough of resource $r$ left to allocate each of them at least a $(1 / n)$ fraction of $r$, as required by SI. Thus, a non-wasteful mechanism satisfying both SI and DPO must allocate, in every step $k$, exactly a $(k / n)$-fraction of some resource and at most a $(k / n)$-fraction of every other resource. In other words, in every step $k$, the mechanism has a pool of available resources - which contains a $(k / n)$-fraction of each resource, minus the fraction already allocated - to be allocated to the $k$ agents that are currently present. The mechanism can only allocate from this pool, and must exhaust at least one resource from the pool.

Example 2 (Understanding Strategyproofness (SP)). In this example, we take a mechanism that may seem SP at first glance, and show that it violates our definition of SP. For simplicity, we will allow the agents to have possibly zero demands for some of the resources in this example. This allows beneficial manipulations for the following simple mechanism, which we call DynAmiC Dictatorship. (We note that DynAmiC DictatorshiP is otherwise strategyproof for strictly positive demands - see the discussion following Theorem 3.) At each step $k$, the mechanism allocates a $1 / n$ share of each resource to agent $k$, takes back the shares of different resources that the agent cannot use, and then allocates resources to the $k$ present agents in the order of their arrival using serial dictatorship, that is, it allocates to each agent as many resources as the agent can use, and then proceeds to the next agent. The mechanism keeps allocating until a $k / n$ share of at least one resource is allocated. Note that the mechanism trivially satisfies SI because it allocates resources as valuable as an equal split to each agent as soon as it arrives. The mechanism would satisfy DPO in our standard setting with non-zero demands, because it is non-wasteful and at every step $k$ it allocates a $k / n$ fraction of at least one resource. Intuitively, it seems that the first agent should not gain by reporting a false demand vector because in each round it gets to pick first and is allowed to take as much as it can use from the available pool of resources. We show that this intuition is incorrect. Let us denote the pool of resources available to the mechanism in any step by a vector of the fraction of each available resource. Consider the case of four agents (agents 1, 2, 3 , and 4$)$, and three resources $\left(R_{1}, R_{2}\right.$, and $\left.R_{3}\right)$. Let the true demand vectors of the agents be as follows:

$$
d_{1}=\langle 1,0.5,0.5\rangle, d_{2}=\langle 0,1,1\rangle, d_{3}=\langle 1,0.5,0\rangle, d_{4}=\langle 0,1,0.5\rangle .
$$

Figure 1 shows the allocations returned by DYNAMIC DICTATORSHIP in various steps when all agents report their true demand vectors. Now, suppose agent 1 raises its demand for $R_{3}$ by reporting a false demand vector $\langle 1,0.5,1\rangle$. In this case the allocations returned by the mechanism in various steps are shown in Figure 2. We can see that the manipulation makes agent 1 strictly worse off in step 2, but strictly better off in the final step. Our definition of SP requires that an agent should not be able to benefit in any step of the process by lying - thus DYNAMIC DICTATORSHIP is not SP.

\subsection{Impossibility Result}

Ideally, we would like to design a dynamic allocation mechanism that is SI, EF, SP, and DPO. However, we show that even satisfying EF and DPO simultaneously is impossible.

Theorem 3. Let $n \geq 3$ and $m \geq 2$. Then no dynamic resource allocation mechanism satisfies $E F$ and $D P O$. 


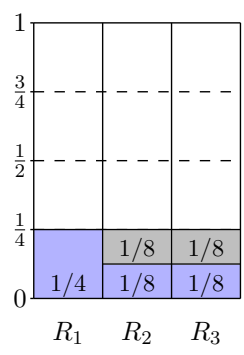

(a) Step 1

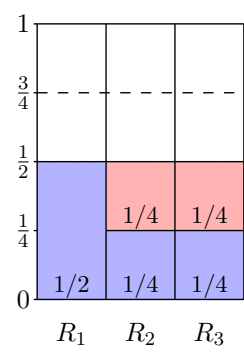

(b) Step 2

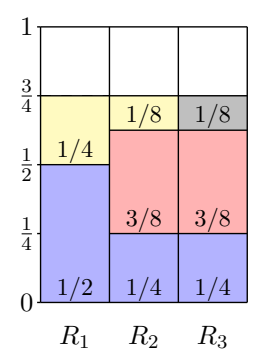

(c) Step 3

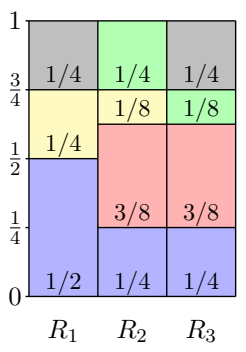

Agent 1

Agent 2

Agent 3

Agent 4

Unallocated resource available in the pool

(d) Step 4

Figure 1: Allocations returned by DyNAMIC DictATORSHIP when agent 1 reports its true demand vector.

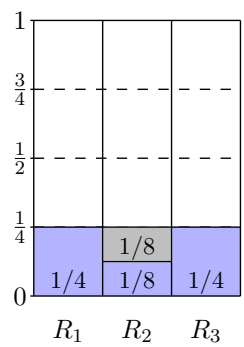

(a) Step 1

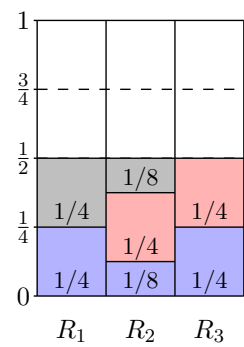

(b) Step 2

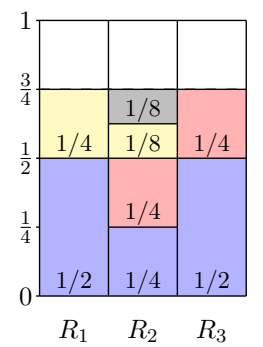

(c) Step 3

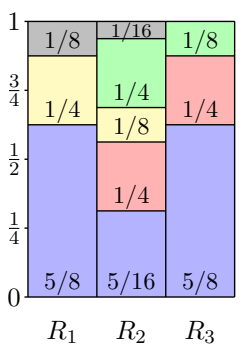

(d) Step 4

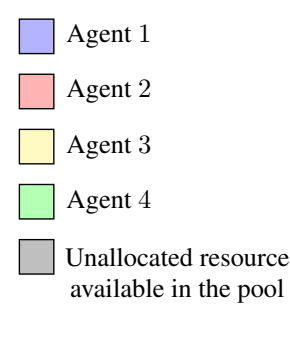

Figure 2: Allocations returned by DYNAMIC DICTATORSHIP when agent 1 manipulates.

Proof. Consider a setting with three agents and two resources. Agents 1 and 2 have demand vectors $\langle 1,1 / 9\rangle$ and $\langle 1 / 9,1\rangle$, respectively (i.e., $d_{11}=1, d_{12}=1 / 9$, etc.). At step 2 (after the second agent arrives), at least one of the two agents must be allocated at least an $x=3 / 5$ share of its dominant resource. Suppose for contradiction that the two agents are allocated $x^{\prime}$ and $x^{\prime \prime}$ shares of their dominant resources where $0<x^{\prime}, x^{\prime \prime}<x$. Then, the total fractions of the two resources allocated at step 2 would be $x^{\prime}+x^{\prime \prime} \cdot(1 / 9)$ and $x^{\prime \prime}+x^{\prime} \cdot(1 / 9)$, both less than $x+x \cdot(1 / 9)=2 / 3$, violating DPO. Without loss of generality, assume that agent 1 is allocated at least an $x=3 / 5$ share of its dominant resource (resource 1) at step 2. If agent 3 reports the demand vector $\langle 1,1 / 9\rangle-$ identical to that of agent 1 - then it can be allocated at most a $2 / 5$ share of its dominant resource (resource 1), and would envy agent 1.

It is easy to extend this argument to the case of $n>3$, by adding $n-3$ agents with demand vectors that are identical to the demand vector of agent 3 . Once again, it can be verified that at the end of step 2, at least one of the first two agents (w.l.o.g., agent 1 ) must be allocated at least a $9 /(5 n)$ share of its dominant resource. If we take the remaining resources (in particular, at most a $1-9 /(5 n)$ share of resource 1$)$, and divide them among the remaining $n-2$ agents that have demand vectors identical to that of agent 1 , at least one of them will get at most a $(1-9 /(5 n)) /(n-2)<9 /(5 n)$ share of its dominant resource, and will envy agent 1 . To extend to the case of $m>2$, let all agents have negligibly small demands for the additional resources. $\square$ (Proof of Theorem 3)

It is interesting to note that if either EF or DPO is dropped, the remaining three axioms can be easily satisfied. For example, the trivial mechanism EQUAL SPLIT that just gives every agent a $1 / n$ share of each resource when it arrives satisfies SI, EF and SP. Achieving SI, DPO, and 
SP is also simple. Indeed, consider the DYNAMIC DiCTATORSHIP mechanism from Example 2. The example explains why DYNAMIC DICTATORSHIP satisfies both SI and DPO. Even though DYNAMIC DICTATORSHIP is not SP under possibly zero demands (as shown in the example), it is clearly SP for strictly positive demands (as assumed throughout this paper). When agent $k$ arrives in step $k$, it is allocated a $1 / n$ share of its dominant resource (and other resources in proportion), and subsequently agent 1 is allocated resources until a $k / n$ share of at least one resource is exhausted. Since every agent requires the exhausted resource due to strictly positive demands, the allocation stops. In summary, all agents except agent 1 receive exactly a $1 / n$ share of their dominant resource when they arrive, and do not receive any resources later on; hence, they cannot gain by reporting a false demand vector. In step $k$, agent 1 receives as much resources as it can from the pool of resources that remain after allocating to agents 2 through $k$ a $1 / n$ share of their dominant resource from an original pool that contains a $k / n$ share of each resource. Therefore, agent 1 also cannot gain from manipulation.

While both EQUAL SPLIT and DYNAMIC DICTATORSHIP satisfy maximal subsets of our proposed desiderata, neither is a compelling mechanism. Since these mechanisms are permitted by dropping EF or DPO entirely, we instead explore relaxations of EF and DPO that rule these mechanisms out and guide us towards more compelling mechanisms.

\section{Relaxing Envy Freeness}

Recall that DPO requires a mechanism to allocate at least a $k / n$ fraction of at least one resource at step $k$, for every $k \in\{1, \ldots, n\}$. Thus the mechanism sometimes needs to allocate a large amount of resources to agents arriving early, potentially making it impossible for the mechanism to prevent the late agents from envying the early agents. In other words, when an agent $i$ enters the system it may envy some agent $j$ that arrived before $i$ did; this is inevitable in order to be able to satisfy DPO. However, it would be unfair to agent $i$ if agent $j$ were allocated more resources since agent $i$ arrived while $i$ still envied $j$. To distill this intuition, we introduce the following dynamic version of EF.

$2^{\prime}$. Dynamic Envy Freeness $(D E F)$. A dynamic allocation mechanism is DEF if at any step an agent $i$ envies an agent $j$ only if $j$ arrived before $i$ did and $j$ has not been allocated any resources since $i$ arrived. Formally, for every $k \in\{1, \ldots, n\}$, if $u_{i}\left(\mathbf{A}_{j}^{k}\right)>u_{i}\left(\mathbf{A}_{i}^{k}\right)$ then $j<i$ and $\mathbf{A}_{j}^{k}=\mathbf{A}_{j}^{i-1}$.

Walsh (2011) studied a dynamic cake cutting setting and proposed forward $E F$, which requires that an agent not envy any agent that arrived later. This notion is weaker than DEF because it does not rule out the case where an agent $i$ envies an agent $j$ that arrived earlier and $j$ received resources since $i$ arrived. In our setting, even the trivial mechanism DYNAMIC DiCTATORSHIP (see Section 3.1) satisfies forward EF, but fails to satisfy our stronger notion of DEF.

We next construct a dynamic resource allocation mechanism - DYNAMIC DRF — that achieves the relaxed fairness notion of DEF, together with SI, DPO, and SP. The mechanism is given as Algorithm 1.

Intuitively, at each step $k$ the mechanism starts from the current allocation among the present agents and keeps allocating resources to agents that have the minimum dominant share at the same rate, until a $k / n$ fraction of at least one resource is allocated. Always allocating to agents that have the minimum dominant share ensures that agents are not allocated any resources while they 


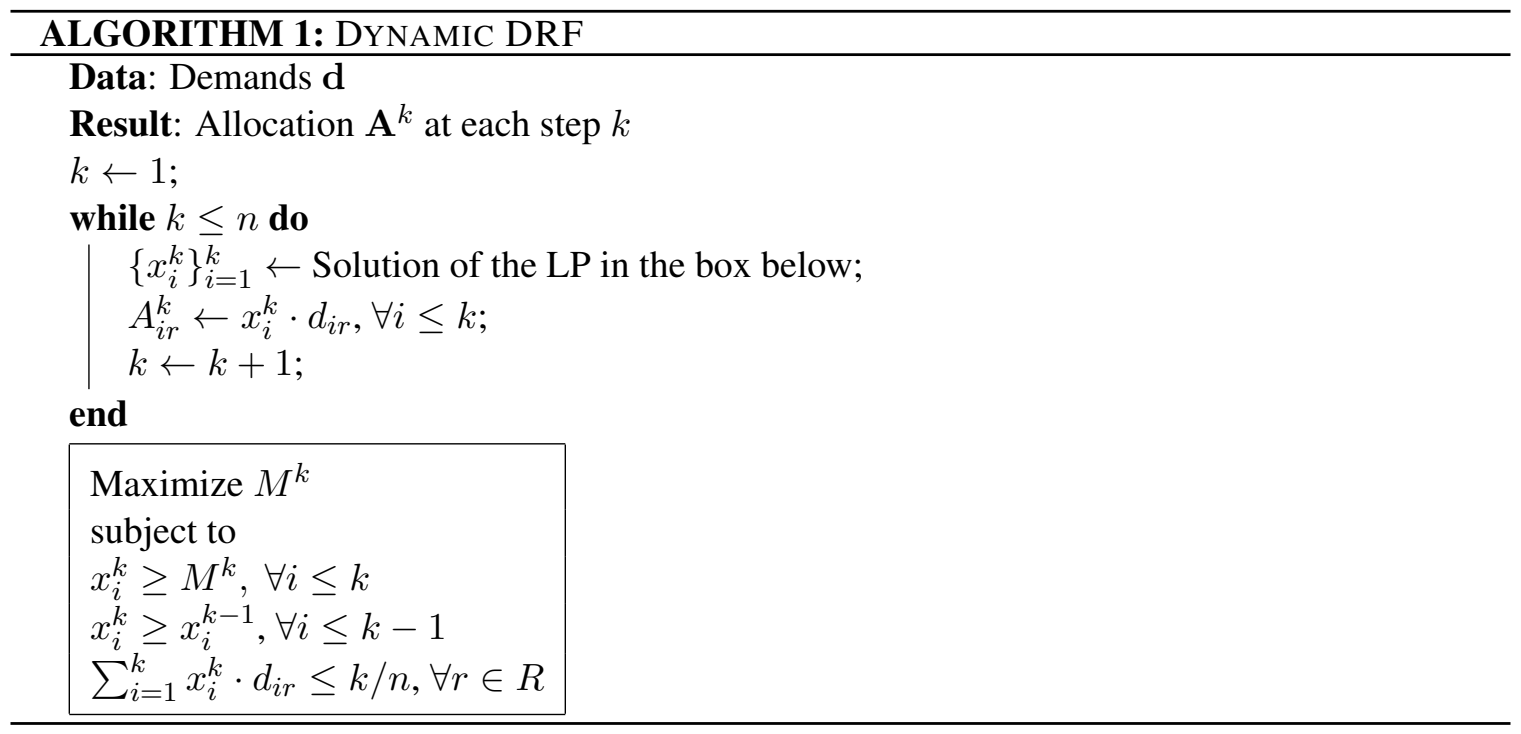

are envied. This water-filling mechanism is a dynamic adaptation of the dominant resource fairness (DRF) mechanism proposed by Ghodsi et. al. (2011). See Figure 3 for an example.

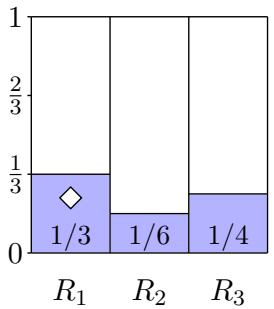

(a) Step 1

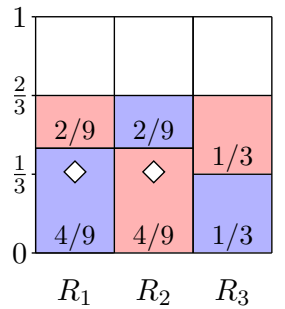

(b) Step 2

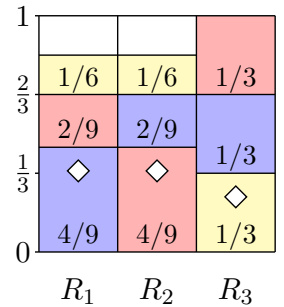

(c) Step 3

Figure 3: Allocations returned by DYNAMIC DRF at various steps for 3 agents with demands $\mathbf{d}_{1}=$ $\langle 1,1 / 2,3 / 4\rangle, \mathbf{d}_{2}=\langle 1 / 2,1,3 / 4\rangle$, and $\mathbf{d}_{3}=\langle 1 / 2,1 / 2,1\rangle$, and three resources $R_{1}, R_{2}$, and $R_{3}$. Agent 1 receives a $1 / 3$ share of its dominant resource at step 1 . At step 2 , water-filling drives the dominant shares of agents 1 and 2 up to $4 / 9$. At step 3 , however, agent 3 can only receive a $1 / 3$ dominant share and the allocations of agents 1 and 2 remain unchanged.

Theorem 4. DYNAMIC DRF satisfies SI, DEF, DPO, and SP, and can be implemented in polynomial time.

Proof. First we show that DYNAMIC DRF satisfies SI. We need to prove that $x_{i}^{k} \geq 1 / n$ for all agents $i \leq k$ at every step $k \in\{1, \ldots, n\}$. We prove this by induction on $k$. For the base case $k=1$, it is easy to see that $x_{1}^{1}=1 / n$ and $M^{1}=1 / n$ is a solution of the LP of DYNAMIC DRF and hence the optimal solution satisfies $x_{1}^{1} \geq M^{1} \geq 1 / n$ (in fact, there is an equality). Assume that this is true at step $k-1$ and let us prove the claim for step $k$, where $k \in\{2, \ldots, n\}$. At step $k$, one feasible solution of the LP is given by $x_{i}^{k}=x_{i}^{k-1}$ for agents $i \leq k-1, x_{k}^{k}=1 / n$ and $M^{k}=1 / n$. To see this, note that it trivially satisfies the first two constraints of the LP, because by the induction 
hypothesis we have $x_{i}^{k-1} \geq 1 / n$ for $i \leq k-1$. Furthermore, in the proposed feasible solution, for any $r \in R$ we have

$$
\sum_{i=1}^{k} x_{i}^{k} \cdot d_{i r}=\sum_{i=1}^{k-1} x_{i}^{k-1} \cdot d_{i r}+\frac{1}{n} \cdot d_{k r} \leq \frac{k-1}{n}+\frac{1}{n} \leq \frac{k}{n},
$$

where the first transition follows from the construction of the feasible solution and the second transition holds because $\left\{x_{i}^{k-1}\right\}_{i=1}^{k-1}$ satisfies the LP of step $k-1$, and in particular the third constraint of the LP. Since a feasible solution achieves $M^{k}=1 / n$, the optimal solution achieves $M^{k} \geq 1 / n$. Thus in the optimal solution $x_{i}^{k} \geq M^{k} \geq 1 / n$ for all $i \leq k$, which is the requirement for SI.

Next we show that DYNAMIC DRF satisfies DPO. Observe that at any step $k$, the third constraint of the LP must be tight for at least one resource in the optimal solution (otherwise every $x_{i}^{k}$ along with $M^{k}$ can be increased by a sufficiently small quantity, contradicting the optimality of $M^{k}$ ). Thus, at each step $k$ the (non-wasteful) mechanism allocates a $k / n$ fraction of at least one resource, which implies that the mechanism satisfies DPO.

To prove that the mechanism satisfies DEF and SP, we first prove several useful lemmas about the allocations returned by the mechanism. In the proof below, $M^{k}$ and $x_{i}^{k}$ refer to the optimal solution of the LP in step $k$. Furthermore, we assume that $x_{i}^{k}=0$ for agents $i>k$ (i.e., agents not present in the system are not allocated any resources). We begin with the following lemma, which essentially shows that if an agent is allocated some resources in a step using water-filling, then the agent's dominant share after the step will be the minimum among the present agents.

Lemma 5. At every step $k \in\{1, \ldots, n\}$, it holds that $x_{i}^{k}=\max \left(M^{k}, x_{i}^{k-1}\right)$ for all agents $i \leq k$.

Proof. Consider any step $k \in\{1, \ldots, n\}$. From the first and the second constraints of the LP it is evident that $x_{i}^{k} \geq M^{k}$ and $x_{i}^{k} \geq x_{i}^{k-1}$ (note that $x_{k}^{k-1}=0$ ), thus $x_{i}^{k} \geq \max \left(M^{k}, x_{i}^{k-1}\right.$ ) for all $i \leq k$. Suppose for contradiction that $x_{i}^{k}>\max \left(M^{k}, x_{i}^{k-1}\right)$ for some $i \leq k$. Then $x_{i}^{k}$ can be reduced by a sufficiently small $\epsilon>0$ without violating any constraints. This makes the third constraint of the LP loose by at least $\epsilon \cdot d_{i r}$, for every resource $r \in R$. Consequently, the values of $x_{j}^{k}$ for $j \neq i$ and $M^{k}$ can be increased by a sufficiently small $\delta>0$ without violating the third constraint of the LP. Finally, $\epsilon$ (and correspondingly $\delta$ ) can be chosen to be small enough so that $x_{i}^{k} \geq M^{k}$ is not violated. It follows that the value of $M^{k}$ can be increased, contradicting the optimality of $M^{k}$. $\square$ (Proof of Lemma 5)

Next we show that at each step $k$, the dominant shares of agents 1 through $k$ are monotonically non-increasing with their time of arrival. This is intuitive because at every step $k$, agent $k$ enters with zero dominant share and subsequently we perform water-filling, hence monotonicity is preserved.

Lemma 6. For all agents $i, j \in N$ such that $i<j$, we have $x_{i}^{k} \geq x_{j}^{k}$ at every step $k \in\{1, \ldots, n\}$.

Proof. Fix any two agents $i, j \in N$ such that $i<j$. We prove the lemma by induction on $k$. The result trivially holds for $k<j$ since $x_{j}^{k}=0$. Assume that $x_{i}^{k-1} \geq x_{j}^{k-1}$ where $k \in\{j, \ldots, n\}$. At step $k$, we have $x_{i}^{k}=\max \left(M^{k}, x_{i}^{k-1}\right) \geq \max \left(M^{k}, x_{j}^{k-1}\right)=x_{j}^{k}$, where the first and the last transition follow from Lemma 5 and the second transition follows from our induction hypothesis. $\square$ (Proof of Lemma 6) 
The following lemma shows that if agent $j$ has a greater dominant share than agent $i$ at some step, then $j$ must have arrived before $i$ and $j$ must not have been allocated any resources since $i$ arrived. Observe that this is very close to the requirement of DEF.

Lemma 7. At any step $k \in\{1, \ldots, n\}$, if $x_{j}^{k}>x_{i}^{k}$ for some agents $i, j \leq k$, then $j<i$ and $x_{j}^{k}=x_{j}^{i-1}$.

Proof. First, note that $j<i$ trivially follows from Lemma 6. Suppose for contradiction that $x_{j}^{k}>x_{j}^{i-1}$ (it cannot be smaller because allocations are irrevocable). Then there exists a step $t \in\{i, \ldots, k\}$ such that $x_{j}^{t}>x_{j}^{t-1}$. Now Lemma 5 implies that $x_{j}^{t}=M^{t} \leq x_{i}^{t}$, where the last transition follows because $x_{i}^{t}$ satisfies the second constraint of the LP at step $t$ (note that $i \leq t$ ). However, $x_{j}^{t} \geq x_{i}^{t}$ due to Lemma 6. Thus, $x_{j}^{t}=x_{i}^{t}$. Now using Lemma $5, x_{j}^{t+1}=\max \left(M^{t+1}, x_{j}^{t}\right)=$ $\max \left(M^{t+1}, x_{i}^{t}\right)=x_{i}^{t+1}$. Extending this argument using a simple induction shows that $x_{j}^{t^{\prime}}=x_{i}^{t^{\prime}}$ for every step $t^{\prime} \geq t$, in particular, $x_{j}^{k}=x_{i}^{k}$, contradicting our assumption. $\square$ (Proof of Lemma 7)

We proceed to show that DYNAMIC DRF satisfies DEF. We need to prove that for any step $k \in$ $\{1, \ldots, n\}$ and any agents $i, j \leq k$, if agent $i$ envies agent $j$ in step $k$ (i.e., $u_{i}\left(\mathbf{A}_{j}^{k}\right)>u_{i}\left(\mathbf{A}_{i}^{k}\right)$ ), then $j<i$ and $x_{j}^{k}=x_{j}^{i-1}$. First, note that $u_{i}\left(\mathbf{A}_{j}^{k}\right)>u_{i}\left(\mathbf{A}_{i}^{k}\right)$ trivially implies that $x_{j}^{k}>x_{i}^{k}$, otherwise for the dominant resource $r_{i}^{*}$ of agent $i$, we would have $A_{i r_{i}^{*}}^{k}=x_{i}^{k} \geq x_{j}^{k} \geq x_{j}^{k} \cdot d_{j r_{i}^{*}}=A_{j r_{i}^{*}}^{k}$ and agent $i$ would not envy agent $j$. Now DEF follows from Lemma 7.

To prove that DYNAMIC DRF is SP, suppose for contradiction that an agent $i \in N$ can report an untruthful demand vector $\mathbf{d}_{i}^{\prime}$ such that the agent is strictly better off in at least one step. Let $k$ be the first such step. Denote by $\hat{x}_{j}^{t}$ the dominant share of an agent $j$ at step $t$ with manipulation (for agent $i$, this is the share of the dominant resource of the untruthful demand vector) and similarly, denote by $\hat{M}^{t}$ the value of $M^{t}$ in the optimal solution of the LP of step $t$ with manipulation.

Lemma 8. $\hat{x}_{j}^{k} \geq x_{j}^{k}$ for every agent $j \leq k$.

Proof. For any agent $j$ such that $x_{j}^{k}>x_{i}^{k}$, we have

$$
x_{j}^{k}=x_{j}^{i-1}=\hat{x}_{j}^{i-1} \leq \hat{x}_{j}^{k} .
$$

Here, the first transition follows from Lemma 7, the second transition holds because manipulation by agent $i$ does not affect the allocation at step $i-1$, and the third transition follows from the LP. For any agent $j$ with $x_{j}^{k} \leq x_{i}^{k}$, we have

$$
x_{j}^{k} \leq x_{i}^{k}<\hat{x}_{i}^{k}=\hat{M}^{k} \leq \hat{x}_{j}^{k} .
$$

The second transition is true because if $\hat{x}_{i}^{k} \leq x_{i}^{k}$ then agent $i$ could not be better off as the true dominant share it receives with manipulation would be no more than it received without manipulation. To justify the third transition, note that agent $i$ must be allocated some resources at step $k$ with manipulation. If $k=i$, this is trivial, and if $k>i$, this follows because otherwise $k$ would not be the first step when agent $i$ is strictly better off as we would have $u_{i}\left(\hat{\mathbf{A}}_{i}^{k-1}\right)=u_{i}\left(\hat{\mathbf{A}}_{i}^{k}\right)>$ $u_{i}\left(\mathbf{A}_{i}^{k}\right) \geq u_{i}\left(\mathbf{A}_{i}^{k-1}\right)$, where $\hat{\mathbf{A}}_{i}^{k}$ denotes the allocation to agent $i$ at step $k$ with manipulation. Thus, $\hat{x}_{i}^{k}>\hat{x}_{i}^{k-1}$, and the third transition now follows from Lemma 5. The last transition holds because $\hat{x}_{j}^{k}$ satisfies the first constraint of the LP of step $k$. Thus, we conclude that $\hat{x}_{j}^{k} \geq x_{j}^{k}$ for all agents $j \leq k . \square$ (Proof of Lemma 8) 
Now, the mechanism satisfies DPO and thus allocates at least a $k / n$ fraction of at least one resource at step $k$ without manipulation. Let $r$ be such a resource. Then the fraction of resource $r$ allocated at step $k$ with manipulation is

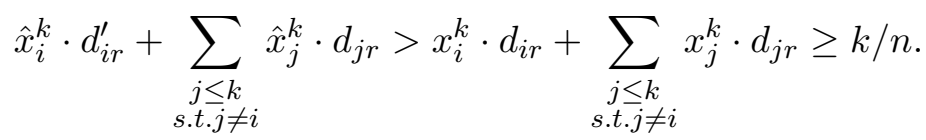

To justify the inequality, note that $\hat{x}_{i}^{k} \cdot d_{i r}^{\prime}>x_{i}^{k} \cdot d_{i r}$ by Equation (1) (as agent $i$ is strictly better off), and in addition $\hat{x}_{j}^{k} \geq x_{j}^{k}$ for every $j \leq k$. However, this shows that more than a $k / n$ fraction of resource $r$ must be allocated at step $k$ with manipulation, which is impossible due to the third constraint of the LP. Hence, a successful manipulation is impossible, that is, DYNAMIC DRF is SP.

Finally, note that the LP has a linear number of variables and constraints, therefore the mechanism can be implemented in polynomial time. $\square$ (Proof of Theorem 4)

\section{Relaxing Dynamic Pareto Optimality}

We saw (Theorem 3) that satisfying EF and DPO is impossible. We then explored an intuitive relaxation of EF. Despite the positive result (Theorem 4), the idea of achieving absolute fairness as conceptualized by $\mathrm{EF}$ - in our dynamic setting is compelling.

As a straw man, consider waiting for all the agents to arrive and then using any EF static allocation mechanism. However, this scheme is highly inefficient, e.g., it is easy to see that one can always allocate each agent at least a $1 / n$ share of its dominant resource (and other resources in proportion) as soon as it arrives and still maintain EF at every step. How much more can be allocated at each step? We put forward a general answer to this question using a relaxed notion of DPO that requires a mechanism to allocate as many resources as possible while ensuring that EF can be achieved in the future, but first we require the following definition. Given a step $k \in\{1, \ldots, n\}$, define an allocation $\mathbf{A}$ over the $k$ present agents with demands $\mathbf{d}_{\leq k}$ to be $E F$-extensible if it can be extended to an EF allocation over all $n$ agents with demands $\mathbf{d}=\left(\mathbf{d}_{\leq k}, \mathbf{d}_{>k}\right)$, for all possible future demand vectors $\mathbf{d}_{>k} \in \mathcal{D}^{n-k}$.

$4^{\prime}$. Cautious Dynamic Pareto optimality (CDPO). A dynamic allocation mechanism satisfies $\mathrm{CDPO}$ if at every step $k$, the allocation $\mathbf{A}^{k}$ returned by the mechanism is not Pareto dominated by any other allocation $\mathbf{A}^{\prime}$ over the same $k$ agents that is EF-extensible.

In other words, a mechanism satisfies CDPO if at every step it selects an allocation that is at least as generous as any allocation that can ultimately guarantee $\mathrm{EF}$, irrespective of future demands.

At first glance, it may not be obvious that CDPO is indeed a relaxation of DPO (i.e., that CDPO is implied by DPO). However, note that DPO requires a mechanism to allocate at least a $k / n$ fraction of at least one resource $r^{*}$ in the allocation $\mathbf{A}^{k}$ at any step $k$, and thus to allocate at least a $1 / n$ fraction of that resource to some agent $i$. Any alternative allocation that Pareto dominates $\mathbf{A}^{k}$ must also allocate at least a $1 / n$ fraction of $r^{*}$ to agent $i$. Consequently, in order to ensure an EF extension over all $n$ agents when all the future demands are identical to the demand of agent $i$, the alternative allocation must allocate at most a $k / n$ fraction of $r^{*}$, as each future agent may also require at least a $1 / n$ fraction of $r^{*}$ to avoid envying agent $i$. It follows that the alternative allocation cannot Pareto dominate $\mathbf{A}^{k}$. Thus, the mechanism satisfies CDPO. 
Recall that DYNAMIC DRF extends the water-filling idea of the static DRF mechanism (Ghodsi et al., 2011) to our dynamic setting. DYNAMIC DRF is unable to satisfy the original EF, because to satisfy DPO - at every step $k$ it needs to allocate resources until a $k / n$ fraction of some resource is allocated. We wish to modify DYNAMIC DRF to focus only on competing with EF-extensible allocations, in a way that achieves CDPO and EF (as well as other properties).

The main technical challenge is checking when an allocation at step $k$ violates EF-extensibility. Indeed, there are uncountably many possibilities for the future demands $\mathbf{d}_{>k}$ over which an EF extension needs to be guaranteed by an EF-extensible allocation! Of course, checking all the possibilities explicitly is not feasible. Ideally, we would like to check only a small number of possibilities. The following lemma establishes that it is sufficient to verify that an EF extension exists under the assumption that all future agents will have the same demand vector that is moreover identical to the demand vector of one of the present agents.

Lemma 9. Let $k$ be the number of present agents, $\mathbf{d}_{\leq k}$ be the demands reported by the present agents, and $\mathbf{A}$ be an EF allocation over the $k$ present agents. Then $\mathbf{A}$ is EF-extensible if and only if there exists an EF extension of $\mathbf{A}$ over all $n$ agents with demands $\mathbf{d}=\left(\mathbf{d}_{\leq k}, \mathbf{d}_{>k}\right)$ for all future demands $\mathbf{d}_{>k} \in \mathcal{D}^{\prime}$, where $\mathcal{D}^{\prime}=\left\{\left\langle\mathbf{d}_{1}\right\rangle^{n-k},\left\langle\mathbf{d}_{2}\right\rangle^{n-k}, \ldots,\left\langle\mathbf{d}_{k}\right\rangle^{n-k}\right\}$.

To prove this lemma, we first introduce the notion of the minimum EF extension. Intuitively, the minimum EF extension is the "smallest" EF extension (allocating the least resources) of a given EF allocation to a larger set of agents. Formally, let $\mathbf{A}$ be an EF allocation over a set of agents $S \subseteq N$ and $\mathbf{A}^{*}$ be an EF extension of $A$ to a set of agents $T \subseteq N(S \subseteq T)$. Then $\mathbf{A}^{*}$ is called the minimum EF extension of $\mathbf{A}$ to $T$ if for any EF extension $\mathbf{A}^{\prime}$ of $\mathbf{A}$ to $T$, we have that $\mathbf{A}^{\prime}$ is an extension of $\mathbf{A}^{*}$. We show that the minimum EF extension exists and exhibits a simple structure.

Lemma 10. Let $\mathbf{A}$ be an EF allocation over a set of agents $S \subseteq N$ and let $x_{i}$ be the dominant share of agent $i \in S$ in $\mathbf{A}$. Let $T$ be such that $S \subseteq T \subseteq N$ and let $\mathbf{A}^{*}$ be an allocation over $T$ with $x_{i}^{*}$ as the dominant share of agent $i \in T$. Let $x_{i}^{*}=x_{i}$ for all $i \in S$, and $x_{i}^{*}=\max _{j \in S} y_{i}^{j}$ for all $i \in T \backslash S$, where $y_{i}^{j}=x_{j} \cdot \min _{r \in R} d_{j r} / d_{i r}$. Then $\mathbf{A}^{*}$ is a minimum EF extension of $\mathbf{A}$ to $T$.

Proof. For agent $i$ with dominant share $x_{i}$ to avoid envying agent $j$ with dominant share $x_{j}$, there must exist $r \in R$ such that $x_{i} \cdot d_{i r} \geq x_{j} \cdot d_{j r}$, that is, $x_{i} \geq x_{j} \cdot d_{j r} / d_{i r}$. It follows that $x_{i} \geq$ $x_{j} \cdot \min _{r \in R} d_{j r} / d_{i r}$, and thus the minimum dominant share is given by $y_{i}^{j}=x_{j} \cdot \min _{r \in R} d_{j r} / d_{i r}$. Now it is easy to argue that any EF extension $\mathbf{A}^{\prime}$ of $\mathbf{A}$ over $T$ must allocate at least an $x_{i}^{*}$ dominant share to any agent $i \in T$, for both $i \in S$ and $i \in T \backslash S$, and thus $\mathbf{A}^{\prime}$ must be an extension of $\mathbf{A}^{*}$.

It remains to prove that $\mathbf{A}^{*}$ is EF. First we prove an intuitive result regarding the minimum dominant share agent $i$ needs to avoid envying agent $j$, namely $y_{i}^{j}$. We claim that for every $r \in R$,

$$
y_{i}^{j} \cdot d_{i r} \leq x_{j} \cdot d_{j r}
$$

Formally, for any $r \in R$,

$$
y_{i}^{j} \cdot d_{i r}=x_{j} \cdot \min _{r^{\prime} \in R} \frac{d_{j r^{\prime}}}{d_{i r^{\prime}}} \cdot d_{i r} \leq x_{j} \cdot \frac{d_{j r}}{d_{i r}} \cdot d_{i r}=x_{j} \cdot d_{j r} .
$$

Therefore, to prevent agent $i$ from envying agent $j$, we need to allocate at least an $x_{j} \cdot d_{j r}$ fraction of resource $r$ to agent $i$ for some $r \in R$. Next we show that $\mathbf{A}^{*}$ is EF, i.e., no agent $i$ envies any agent $j$ in $\mathbf{A}^{*}$. We consider four cases. 
Case 1: $i \in S$ and $j \in S$. This case is trivial as $\mathbf{A}^{*}$ is identical to $\mathbf{A}$ over $S$ and $\mathbf{A}$ is EF.

Case 2: $i \in T \backslash S$ and $j \in S$. This case is also trivial because $i$ receives at least a $y_{i}^{j}$ fraction of its dominant resource.

Case 3: $i \in S$ and $j \in T \backslash S$. We must have $x_{j}=y_{j}^{t}$ for some $t \in S$. Agent $i$ does not envy agent $t$ in $\mathbf{A}$, and hence in $\mathbf{A}^{*}$. Thus, there exists a resource $r \in R$ such that $A_{i r}^{*} \geq A_{t r}^{*} \geq A_{j r}^{*}$, where the last step follows from Equation (2). Thus, agent $i$ does not envy agent $j$.

Case 4: $i \in T \backslash S$ and $j \in T \backslash S$. Similarly to Case 3, let $x_{j}=y_{j}^{t}$ for some $t \in S$. Now $x_{i} \geq y_{i}^{t}$, so agent $i$ does not envy agent $t$ in $\mathbf{A}^{*}$. Thus, there exists a resource $r$ such that $A_{i r}^{*} \geq A_{t r}^{*} \geq A_{j r}^{*}$, where again the last step follows from Equation (2).

Therefore, $\mathbf{A}^{*}$ is an EF extension of $\mathbf{A}$ over $T$ and we have already established that any EF extension of $\mathbf{A}$ over $T$ must be an extension of $\mathbf{A}^{*}$. We conclude that $\mathbf{A}^{*}$ is a minimum EF extension of $\mathbf{A}$ over T. $\square$ (Proof of Lemma 10)

It is not hard to see from the construction of the minimum EF extension that it not only exists, it is unique. We are now ready to prove Lemma 9.

Proof of Lemma 9. The "only if" direction of the proof is trivial. To prove the "if" part, we prove its contrapositive. Assume that there exist future demand vectors $\hat{\mathbf{d}}_{>k} \in \mathcal{D}^{n-k}$ such that there does not exist any EF extension of $\mathbf{A}$ to $N$ with demands $\hat{\mathbf{d}}=\left(\mathbf{d}_{\leq k}, \hat{\mathbf{d}}_{>k}\right)$. We want to show that there exists $\mathbf{d}_{>k}^{\prime} \in \mathcal{D}^{\prime}$ for which there is no EF extension as well.

Let $K=\{1, \ldots, k\}$ and $N \backslash K=\{k+1, \ldots, n\}$. Denote the minimum EF extension of $\mathbf{A}$ to $N$ with demands $\hat{\mathbf{d}}$ by $\mathbf{A}^{*}$. Let the dominant share of agent $i \in K$ in $\mathbf{A}$ be $x_{i}$ and the dominant share of agent $j \in N$ in $\mathbf{A}^{*}$ be $x_{j}^{*}$.

No EF extension of $\mathbf{A}$ over $N$ with demands $\hat{\mathbf{d}}$ is feasible, hence $\mathbf{A}^{*}$ must be infeasible too. Therefore, there exists a resource $r$ such that $\sum_{i=1}^{n} x_{i}^{*} \cdot d_{i r}>1$. Note that for every agent $j \in N \backslash K$, there exists an agent $i \in K$ such that $x_{j}^{*}=x_{i} \cdot \min _{r^{\prime} \in R} d_{i r^{\prime}} / d_{j r^{\prime}}$, and hence $x_{j}^{*} \cdot d_{j r} \leq x_{i} \cdot d_{i r}$ by Equation (2). Taking the maximum over $i \in K$, we get that $x_{j}^{*} \cdot d_{j r} \leq \max _{i \in K}\left(x_{i} \cdot d_{i r}\right)$ for every agent $j \in N \backslash K$. Taking $t \in \arg \max _{i \in K}\left(x_{i} \cdot d_{i r}\right)$,

$$
\begin{aligned}
1<\sum_{i=1}^{n} x_{i}^{*} \cdot d_{i r} & =\sum_{i=1}^{k} x_{i}^{*} \cdot d_{i r}+\sum_{i=k+1}^{n} x_{i}^{*} \cdot d_{i r} \\
& \leq \sum_{i=1}^{k} x_{i} \cdot d_{i r}+(n-k) \cdot x_{t} \cdot d_{t r} .
\end{aligned}
$$

Consider the case where $\mathbf{d}_{>k}^{\prime}=\left\langle\mathbf{d}_{t}\right\rangle^{n-k} \in \mathcal{D}^{\prime}$. The minimum EF extension $\mathbf{A}^{\prime}$ of $\mathbf{A}$ to $N$ with demands $\mathbf{d}^{\prime}=\left\langle\mathbf{d}_{\leq k}, \mathbf{d}_{>k}^{\prime}\right\rangle$ allocates an $x_{i}$ dominant share to every $i \in K$ (same as $\mathbf{A}$ ) and allocates exactly an $x_{t}$ dominant share to every $j \in N \backslash K$. Thus, the fraction of resource $r$ allocated in $\mathbf{A}^{\prime}$ is $\sum_{i=1}^{k} x_{i} \cdot d_{i r}+(n-k) \cdot x_{t} \cdot d_{t r}>1$, implying that the minimum EF extension of $\mathbf{d}_{>k}^{\prime}$ is infeasible. We conclude that there is no feasible EF extension for $\mathbf{d}_{>k}^{\prime}$, as required. $\square$ (Proof of Lemma 9)

The equivalent condition of Lemma 9 provides us with $k \cdot m$ linear constraints that can be checked to determine whether an allocation over $k$ agents is EF-extensible. Using this machinery, we can write down a "small" linear program (LP) that begins with the allocation chosen in the previous step (recall that the allocations are irrevocable), gives agent $k$ a jump start so that it does not envy agents 1 through $k-1$, and then uses water-filling to allocate resources similarly to 
DYNAMIC DRF, but subject to the constraint that the allocation stays EF-extensible. This intuition is formalized via the mechanism CAUTIOUS LP, which is given as Algorithm 2.

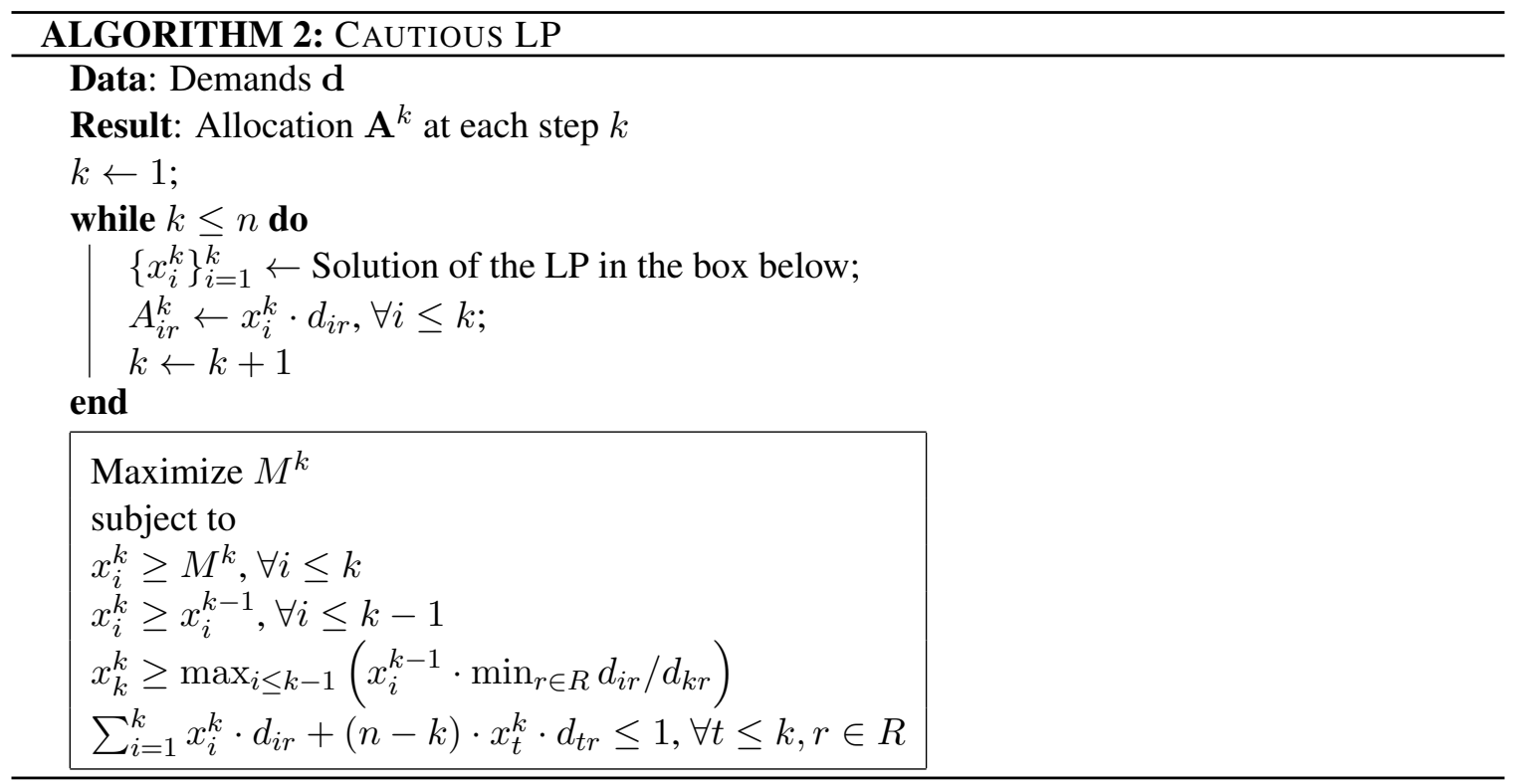

The mechanism's third LP constraint jump-starts agent $k$ to a level where it does not envy earlier agents, and the fourth LP constraint is derived from Lemma 9. To see why the mechanism satisfies CDPO, observe that if at any step $k$ there is an EF-extensible allocation $\mathbf{A}^{\prime}$ that Pareto dominates the allocation $\mathbf{A}^{k}$ returned by the mechanism, then (by Lemma 9) $\mathbf{A}^{\prime}$ must also satisfy the LP at step $k$. However, it can be shown that no allocation from the feasible region of the LP can Pareto dominate $\mathbf{A}^{k}$. Indeed, if an allocation from the feasible region did dominate $\mathbf{A}^{k}$, we could redistribute some of the resources of the agent that is strictly better off to obtain a feasible allocation with a value of $M^{k}$ that is higher than the optimal solution. It is also easy to see why intuitively CAUTIOUS LP is $\mathrm{EF}$ : the initial allocation to agent $k$ achieves an EF allocation over the $k$ agents, and water-filling preserves EF because it always allocates to agents with minimum dominant share. It is equally straightforward to show that CAUTIOUS LP also satisfies SI. Establishing SP requires some work, but the proof is mainly a modification of the proof of Theorem 4 . We are therefore able to establish the following theorem, which formalizes the guarantees given by CAUTIOUS LP.

Theorem 11. CAUTIOUS LP satisfies SI, EF, CDPO, and SP, and can be implemented in polynomial time.

Proof. The proof is along the lines of the proof of Theorem 4. For now, assume that the LP is feasible at each step and thus the mechanism does return an allocation at each step (we show this below). In the LP at step $k$, let

$$
E^{k}=\max _{i \leq k-1}\left(x_{i}^{k-1} \cdot \min _{r \in R} d_{i r} / d_{k r}\right) .
$$

Intuitively, $E^{k}$ is the jump start that agent $k$ requires at the beginning of step $k$ to be envy free of the allocations of agents 1 through $k-1$ from step $k-1$. 
Proof of CDPO: First we show that CAUTIOUs LP satisfies CDPO. Assume for contradiction, that at some step $k \in\{1, \ldots, n\}$, an alternative EF-extensible allocation $\mathbf{A}^{\prime}$ over the $k$ present agents Pareto dominates the allocation $\mathbf{A}^{k}$ returned by the mechanism. Let $x_{i}^{\prime}$ be the dominant share of agent $i$ in $\mathbf{A}^{\prime}$, for $i \leq k$. Since $\mathbf{A}^{\prime}$ Pareto dominates $\mathbf{A}^{k}$, we have that $x_{i}^{\prime} \geq x_{i}^{k}$ for every $i \leq k$. This trivially implies that $\mathbf{A}^{\prime}$ also satisfies the first three constraints of the LP at step $k$. Moreover, since $\mathbf{A}^{\prime}$ is EF-extensible, it also satisfies the fourth constraint of the LP at step $k$ as the fourth constraint only requires EF extension to exist in specific cases (in particular, it requires the minimum EF extension and thus any EF extension over all $n$ agents to exist when all future demand vectors are identical to the demand vector of some present agent). Thus, $\mathbf{A}^{\prime}$ is in the feasible region of the LP and Pareto dominates an optimal solution $\mathbf{A}^{k}$. Now, taking back the extra resources that $\mathbf{A}^{\prime}$ allocates to agents compared to $\mathbf{A}^{k}$ shows that the fourth constraint is not tight in $\mathbf{A}^{k}$ for any value of $t$ and $r$ (the assumption of strictly positive demands is crucial here). However, this implies that in the allocation $\mathbf{A}^{k}$, every $x_{i}^{k}$ and correspondingly $M^{k}$ can be increased by a sufficiently small quantity while still satisfying the LP at step $k$, which contradicts the optimality of $\mathbf{A}^{k}$. Thus, no alternative EF-extensible allocation can Pareto dominate the allocation given by the mechanism at any step, i.e., CAUTIOUS LP satisfies CDPO.

Proof of SI: Next, we show that CAUTIOUS LP satisfies SI. We show this by induction over step $k$. For the base case $k=1$, it is easy to show that setting $x_{1}^{1}=1 / n$ and $M^{k}=1 / n$ satisfies the LP at step 1; it trivially satisfies the first three constraints of the LP and for the fourth constraint, observe that

$$
\frac{1}{n} \cdot d_{i r}+(n-1) \cdot \frac{1}{n} \cdot d_{i r}=d_{i r} \leq 1, \forall r \in R .
$$

Therefore, in the optimal solution, $M^{1} \geq 1 / n$ and thus $x_{1}^{1} \geq 1 / n$ (in fact, equality holds).

Now consider any step $k \in\{2, \ldots, n\}$. As our induction hypothesis, we assume that $x_{i}^{t} \geq 1 / n$ for all agents $i \leq t$, at every step $t \leq k-1$. We want to show that $x_{i}^{k} \geq 1 / n$ for all agents $i \leq k$. Consider two cases.

1. $E^{k} \geq 1 / n$. Observe that $x_{i}^{k-1} \geq 1 / n$ for all $i \leq k-1$ due to the induction hypothesis. Thus, using the second and the third constraints of the LP at step $k$, we have $x_{i}^{k} \geq 1 / n$ for all $i \leq k$.

2. $E^{k}<1 / n$. We first show that $x_{i}^{k}=x_{i}^{k-1}$ for $i \leq k-1, x_{k}^{k}=1 / n$ and $M^{k}=1 / n$ is in the feasible region of the LP at step $k$. Note that this assignment trivially satisfies the first three constraints of the LP.

For the fourth constraint, fix any $r \in R$. Define $T_{r}=\max _{i \leq k-1} x_{i}^{k-1} \cdot d_{i r}$. First, we show that $\sum_{i=1}^{k-1} x_{i}^{k-1} \cdot d_{i r} \leq 1-(n-k+1) \cdot \max \left(T_{r}, 1 / n\right)$. To see this, note that $\left\{x_{i}^{k-1}\right\}_{i=1}^{k-1}$ satisfies the LP at step $k-1$ and, in particular, the fourth constraint of the LP. Therefore,

$$
\sum_{i=1}^{k-1} x_{i}^{k-1} \cdot d_{i r}+(n-k+1) \cdot T_{r} \leq 1 \Longrightarrow \sum_{i=1}^{k-1} x_{i}^{k-1} \cdot d_{i r} \leq 1-(n-k+1) \cdot T_{r} \text {. }
$$

Now we prove that

$$
\sum_{i=1}^{k-1} x_{i}^{k-1} \cdot d_{i r} \leq 1-(n-k+1) \cdot 1 / n=(k-1) / n .
$$


Suppose for contradiction that the left hand side is more than $(k-1) / n$. Then, by the pigeonhole principle, there exists some agent $i \leq k-1$ such that $x_{i}^{k-1} \cdot d_{i r} \geq 1 / n$, and thus $T_{r} \geq 1 / n$. But we have already shown that

$$
\sum_{i=1}^{k-1} x_{i}^{k-1} \cdot d_{i r} \leq 1-(n-k+1) \cdot T_{r} \leq 1-(n-k+1) \cdot 1 / n=(k-1) / n
$$

contradicting our assumption; this establishes (3). Thus, we have that

$$
\sum_{i=1}^{k-1} x_{i}^{k-1} \cdot d_{i r} \leq 1-(n-k+1) \cdot \max \left(T_{r}, \frac{1}{n}\right) .
$$

Finally, we show that in the fourth constraint of the LP, $x_{t}^{k} \cdot d_{t r} \leq \max \left(T_{r}, 1 / n\right)$. To see this, observe that for $t \leq k-1, x_{t}^{k} \cdot d_{t r}=x_{t}^{k-1} \cdot d_{t r} \leq T_{r}$ and for $t=k, x_{t}^{k} \cdot d_{t r}=1 / n \cdot d_{k r} \leq 1 / n$. Thus, the fourth constraint of the LP is satisfied for every $t \leq k$ and every $r \in R$.

We have established that CAUTIOUS LP satisfies SI. Our next goal is to prove that the mechanism also satisfies EF and SP. As in the proof of Theorem 4, we first establish several useful lemmas about the allocations returned by CAUTIOUS LP. In the proof below, $M^{k}$ and $x_{i}^{k}$ refer to the optimal solution of the LP in step $k$.

We begin with the following lemma (similar to Lemma 5), which essentially shows that if an agent is allocated some resources in step $k$ using water-filling (in addition to the jump-start to $E^{k}$ for agent $k$ ), then the agent's dominant share after the step would be the minimum among the present agents.

Lemma 12. At every step $k \in\{1, \ldots, n\}$, it holds that $x_{i}^{k}=\max \left(M^{k}, x_{i}^{k-1}\right)$ for all agents $i \leq k-1$, and $x_{k}^{k}=\max \left(M^{k}, E^{k}\right)$.

Proof. Consider any step $k \in\{1, \ldots, n\}$. From the first three constraints of the LP, it is evident that $x_{i}^{k} \geq M^{k}$ for all $i \leq k, x_{i}^{k} \geq x_{i}^{k-1}$ for all $i \leq k-1$ and $x_{k}^{k} \geq E^{k}$. Thus, $x_{i}^{k} \geq \max \left(M^{k}, x_{i}^{k-1}\right)$ for all $i \leq k-1$ and $x_{k}^{k} \geq \max \left(M^{k}, E^{k}\right)$.

Suppose for contradiction that a strict inequality holds for some agent $i \leq k$. Then $x_{i}^{k}$ can be reduced by a sufficiently small $\epsilon>0$ without violating any constraints. This makes the third constraint of the LP loose by at least $\epsilon \cdot d_{i r}$, for every resource $r \in R$. Consequently, the values of $x_{j}^{k}$ for $j \neq i$ and $M^{k}$ can be increased by a sufficiently small $\delta>0$ without violating the third constraint of the LP. Finally, $\epsilon$ (and correspondingly $\delta$ ) can be chosen to be small enough so that $x_{i}^{k} \geq M^{k}$ is not violated. It follows that the value of $M^{k}$ can be increased, contradicting the optimality of $M^{k} . \square$ (Proof of Lemma 12)

Next, we formulate the equivalent of Lemma 6 as two separate lemmas. First we show that if an agent has greater or equal dominant share than another agent in some step (where both are present), then the order is preserved in future steps. Next we show that at each step $k$, the dominant shares of agents 1 through $k$ are monotonically non-increasing with their time of arrival, except for agents that have not received any resources apart from their jump-start.

Lemma 13. For any agents $i, j \in N$ and any step $k \geq \max (i, j)$ (i.e., both agents are present at step $k$ ), $x_{i}^{k} \geq x_{j}^{k}$ implies that $x_{i}^{t} \geq x_{j}^{t}$ for all $t \geq k$. 
Proof. Fix any two agents $i, j \in N$ and step $k \geq \max (i, j)$ such that $x_{i}^{k} \geq x_{j}^{k}$. We use induction on $t$. The result trivially holds for $t=k$. Consider any $t>k$ and assume the result holds for step $t-1$. Then, since $t>k \geq \max (i, j)$ we know that $x_{i}^{t}=\max \left(x_{i}^{t-1}, M^{t}\right) \geq \max \left(x_{j}^{t-1}, M^{t}\right)=x_{j}^{t}$, where the first and the last transitions follow from Lemma 12 and the second transition follows from our induction hypothesis. $\square$ (Proof of Lemma 13)

Lemma 14. For all agents $i, j \in N$ such that $i<j$ and any step $k \geq j$, we have that either $i$ ) $x_{i}^{k} \geq x_{j}^{k}$ or ii) $x_{j}^{k}=x_{j}^{j}=E^{j}$.

Proof. Fix any two agents $i, j \in N$ such that $i<j$ and any step $k \geq j$. Note that $x_{j}^{k} \geq x_{j}^{j} \geq$ $E^{j}$, where the first inequality is due to irrevocability of resources and the last inequality is due to Lemma 12. If $x_{j}^{k}=E^{j}$, then the lemma trivially holds. Assume $x_{j}^{k}>E^{j}$. Consider the first step $t$ where $x_{j}^{t}>E^{j}$ (thus $j \leq t \leq k$ ). If $t=j$, then we have $x_{j}^{j}>E^{j}$. If $t>j$, then we have $x_{j}^{t}>x_{j}^{t-1}$ since $x_{j}^{t-1}=E^{j}$ by the definition of $t$. In any case, Lemma 12 implies that $x_{j}^{t}=M^{t} \leq x_{i}^{t}$. Thus we have $x_{j}^{t} \leq x_{i}^{t}$ and now Lemma 13 implies that $x_{j}^{k} \leq x_{i}^{k}$. $\square$ (Proof of Lemma 14)

We now consider the equivalent of Lemma 7 from the proof of Theorem 4, and observe that there are two cases. If agent $j$ has greater dominant share than agent $i$ at some step, then either $j$ arrived before $i$ and $j$ has not been allocated any resources since $i$ arrived (as we had previously), or $j$ arrived after $i$ and has not been allocated any resources apart from its jump-start.

Lemma 15. For any agents $i, j \in N$ and any step $k \geq \max (i, j)$ (i.e., both agents are present), $x_{j}^{k}>x_{i}^{k}$ implies that either $\left.i\right) j<i$ and $x_{j}^{k}=x_{j}^{i-1}$, or ii) $j>i$ and $x_{j}^{k}=x_{j}^{j}=E^{j}$.

Proof. Fix any two agents $i, j \in N$ and any step $k \geq \max (i, j)$ such that $x_{j}^{k}>x_{i}^{k}$. Note that if $j>i$ then Lemma 14 implies that $x_{j}^{k}=x_{j}^{j}=E^{j}$ and the result holds trivially. Now assume $j<i$.

Suppose for contradiction that $x_{j}^{k}>x_{j}^{i-1}$ (it cannot be smaller because allocations are irrevocable). Then there exists a step $t \in\{i, \ldots, k\}$ such that $x_{j}^{t}>x_{j}^{t-1}$. Therefore, Lemma 12 implies that $x_{j}^{t}=M^{t} \leq x_{i}^{t}$. Using Lemma 13 this shows that $x_{j}^{k} \leq x_{i}^{k}$, which is a contradiction to the assumption that $x_{j}^{k}>x_{i}^{k}$. Thus we have $x_{j}^{k}=x_{j}^{i-1}$, as required. $\square$ (Proof of Lemma 15)

Finally, we establish an additional lemma which will be helpful in proving SP. For agents $i, j$ such that $j>i$, if the jump-start $E^{j}$ for agent $j$ requires allocating agent $j$ greater dominant share than agent $i$ had in step $j-1$, then clearly the jump-start must have been due to agent $j$ envying some agent $l \neq i$, and $l$ must have greater dominant share than $i$ in step $j-1$. But then using Lemma 14 and extending the argument, we can eventually trace this back to an agent $t<i$. We show that we can find such $t<i$ such that the jump-start of the original agent $j$ was actually due to agent $j$ envying agent $t$.

Lemma 16. For any agents $i, j \in N$ such that $j>i, E^{j}>x_{i}^{j}$ implies that $E^{j}=x_{t}^{j-1}$. $\min _{r \in R} d_{t r} / d_{j r}$, for some agent $t<i$.

Proof. Fix any agent $i \in N$. We use induction over $j \in\{i+1, \ldots, n\}$. First, we prove several implications that hold for any agent $j>i$. Recall that $E^{j}=\max _{p<j}\left(x_{p}^{j-1} \cdot \min _{r \in R} d_{p r} / d_{j r}\right)$. 
Thus, we have $E^{j}=x_{l}^{j-1} \cdot \min _{r \in R} d_{l r} / d_{j r}$ for some agent $l<j$. But it does not follow from the definition that we can take $l<i$. Observe that

$$
x_{l}^{j-1} \geq x_{l}^{j-1} \cdot \min _{r \in R} d_{t r} / d_{j r}=E^{j}>x_{i}^{j} \geq x_{i}^{j-1},
$$

where the first transition holds since $\min _{r \in R} d_{t r} / d_{j r}$ is at most 1 (consider the dominant resource of agent $j$ ), the third transition is the assumption of the lemma and the last transition holds since allocations are irrevocable.

Now we have three cases. If $l<i$, then we are done. Further, $l \neq i$ since Equation (4) shows that $x_{l}^{j-1}>x_{i}^{j-1}$. Now assume that $l>i$. Note that this case cannot appear in the base case $j=i+1$ since $l<j$. Therefore, the argument given above already shows that the lemma holds for the base case $j=i+1$. By our induction hypothesis, we assume that the lemma holds for agent $l<j$. Now since $l>i$ and $x_{l}^{j-1}>x_{i}^{j-1}$, Lemma 15 implies that $x_{l}^{j-1}=x_{l}^{l}=E^{l}$ and thus $E^{l}>x_{i}^{j-1} \geq x_{i}^{l}$ where $x_{i}^{j-1} \geq x_{i}^{l}$ because $l<j$ and allocations are irrevocable. Due to our induction hypothesis, there exists $t<i$ such that $E^{l}=x_{t}^{l-1} \cdot \min _{r \in R} d_{t r} / d_{l r}$. We prove that $E^{j}=x_{t}^{j-1} \cdot \min _{r \in R} d_{t r} / d_{j r}$. Indeed,

$$
\begin{aligned}
E^{j} & =x_{l}^{j-1} \cdot \min _{r \in R} d_{l r} / d_{j r} \\
& =E^{l} \cdot \min _{r \in R} d_{l r} / d_{j r} \\
& =x_{t}^{l-1} \cdot \min _{r \in R} d_{t r} / d_{l r} \cdot \min _{r \in R} d_{l r} / d_{j r} \\
& \leq x_{t}^{l-1} \cdot \min _{r \in R} d_{t r} / d_{j r} \\
& \leq x_{t}^{j-1} \cdot \min _{r \in R} d_{t r} / d_{j r} \leq E^{j} .
\end{aligned}
$$

Here, the fourth transition is true because for any $r^{\prime} \in R$,

$$
\frac{d_{t r^{\prime}}}{d_{j r^{\prime}}}=\frac{d_{t r^{\prime}}}{d_{l r^{\prime}}} \cdot \frac{d_{l r^{\prime}}}{d_{j r^{\prime}}} \geq \min _{r \in R} \frac{d_{t r}}{d_{l r}} \cdot \min _{r \in R} \frac{d_{l r}}{d_{j r}} .
$$

Taking minimum over all $r^{\prime} \in R$, we get that $\min _{r \in R} d_{t r} / d_{j r} \geq \min _{r \in R} d_{t r} / d_{l r} \cdot \min _{r \in R} d_{l r} / d_{j r}$. The last transition holds due to the definition of $E^{j}$. Now it is trivial to see that we must have equality at every step, so $E^{j}=x_{t}^{j-1} \cdot \min _{r \in R} d_{t r} / d_{j r}$ for $t<i$, as required. $\square$ (Proof of Lemma 16)

Proof of LP Feasibility and EF: Now we use an inductive argument to simultaneously show that the LP of CAUTIOUS LP is feasible at every step and that CAUTIOUS LP satisfies EF. Consider the following induction hypothesis: the LP at step $t$ is feasible and the allocation $\mathbf{A}^{t}$ returned by the mechanism at step $t$ is EF. For the base case $t=1$, the LP is trivially feasible and the allocation $\mathbf{A}^{1}$ is also trivially EF. Assume that the hypothesis holds for $t=k-1$ for some step $k \in\{2, \ldots, n\}$. We want to show that the hypothesis holds for step $k$.

For feasibility, we show that the allocation $\mathbf{A}^{*}$ given by $x_{i}^{k}=x_{i}^{k-1}$ for $i \leq k-1$ and $x_{k}^{k}=E^{k}$ along with $M^{k}=0$ satisfies the LP at step $k$. Clearly, it satisfies the first three constraints of the LP. To see why it satisfies the fourth constraint, note that $\mathbf{A}^{k-1}$ is an EF allocation due to our induction hypothesis. Moreover, it satisfies the LP at step $k-1$, in particular, the fourth constraint of the LP. Hence Lemma 9 implies that $\mathbf{A}^{k-1}$ must be an EF-extensible allocation. Let $\mathbf{d}_{k}$ denote the 
demand reported by agent $k$ in step $k$ and let $\mathbf{d}_{>k} \in \mathcal{D}^{n-k}$. Then any EF extension of $\mathbf{A}^{k-1}$ over all $n$ agents with future demands $\left(\mathbf{d}_{k}, \mathbf{d}_{>k}\right)$ is an EF extension of $\mathbf{A}^{*}$ over all $n$ agents with future demands $\mathbf{d}_{>k}$. Since this holds for any $\mathbf{d}_{>k} \in \mathcal{D}^{n-k}, \mathbf{A}^{*}$ is EF-extensible and hence satisfies the fourth constraint of the LP. We conclude that the LP is feasible at step $k$.

Now we want to show that the allocation $\mathbf{A}^{k}$ is an EF allocation. Intuitively, we can see that the mechanism starts from $\mathbf{A}^{*}$ which is $\mathrm{EF}$ (it is a minimum EF extension), and then uses waterfilling to allocate more resources in a way that preserves EF. Formally, note that the dominant shares allocated to agents in $\mathbf{A}^{k}$ are given by Lemma 12. Take any two agents $i, j \leq k$. We want to show that agent $i$ does not envy agent $j$ in step $k$. Denote the dominant share of an agent $l$ in $\mathbf{A}^{*}$ by $x_{l}^{*}$, i.e., $x_{l}^{*}=x_{l}^{k-1}$ for $l \leq k-1$ and $x_{k}^{*}=E^{k}$. It holds that

$$
\begin{aligned}
x_{i}^{k} & =\max \left(x_{i}^{*}, M^{k}\right) \geq \max \left(x_{j}^{*} \cdot \min _{r \in R} \frac{d_{j r}}{d_{i r}}, M^{k}\right) \geq \max \left(x_{j}^{*}, M^{k}\right) \cdot \min _{r \in R} \frac{d_{j r}}{d_{i r}} \\
& =x_{j}^{k} \cdot \min _{r \in R} d_{j r} / d_{i r},
\end{aligned}
$$

where the first and the last transitions follow from Lemma 12, the second transition holds since the allocation $\mathbf{A}^{*}$ is $\mathbf{E F}$, and the third transition holds since the quantity $\min _{r \in R} d_{j r} / d_{i r}$ is at most 1 . Thus, $\mathbf{A}^{k}$ is EF. By induction, it holds that the LP of CAUTIOUs LP is feasible at every step and Cautious LP is EF.

Proof of SP: Our last task is to prove that CAUTIOUS LP is SP. Suppose for contradiction that an agent $i \in N$ can report an untruthful demand vector $\mathbf{d}_{i}^{\prime}$ such that the agent is strictly better off in at least one step. Let $k$ be the first such step. Denote by $\hat{x}_{j}^{t}$ the dominant share of an agent $j$ at step $t$ with manipulation (for agent $i$, this is the share of the dominant resource of the untruthful demand vector) and, similarly, denote by $\hat{M}^{t}$ the value of $M^{t}$ in the optimal solution of the LP of step $t$ with manipulation.

Lemma 17. $\hat{x}_{j}^{k} \geq x_{j}^{k}$ for every agent $j \leq k$.

Proof. Fix any agent $j \leq k$. We provide a case by case analysis and show that the lemma holds in each case.

1. $x_{j}^{k} \leq x_{i}^{k}$. In this case, we have

$$
x_{j}^{k} \leq x_{i}^{k}<\hat{x}_{i}^{k}=\hat{M}^{k} \leq \hat{x}_{j}^{k}
$$

The second transition holds because if $\hat{x}_{i}^{k} \leq x_{i}^{k}$ then agent $i$ could not be better off as the share of the dominant resource of its true demand vector that it receives with manipulation would be no more than it received without manipulation. To justify the third transition, note that agent $i$ must be allocated some resources at step $k$ with manipulation. If $k=i$, then note that since $E^{i}$ only depends on the allocation at step $i-1$ which is not affected due to manipulation by agent $i$, we have $\hat{E}^{i}=E^{i} \leq x_{i}^{i}<\hat{x}_{i}^{i}$ and Lemma 12 implies that $\hat{x}_{i}^{i}=\hat{M}^{i}$. If $k>i$ and $\hat{x}_{i}^{k} \neq \hat{M}^{k}$, then Lemma 12 implies that $\hat{x}_{i}^{k}=\hat{x}_{i}^{k-1}$, but then $u_{i}\left(\hat{\mathbf{A}}_{i}^{k-1}\right)=u_{i}\left(\hat{\mathbf{A}}_{i}^{k}\right)>u_{i}\left(\mathbf{A}_{i}^{k}\right) \geq u_{i}\left(\mathbf{A}_{i}^{k-1}\right)$, where $\hat{\mathbf{A}}_{i}^{k}$ is the allocation to agent $i$ at step $k$ with manipulation. That is, agent $i$ would have been better off with manipulation in step $k-1$, which is a contradiction since $k$ is the first such step. The last transition holds because $\hat{x}_{j}^{k}$ satisfies the first constraint of the LP of step $k$ with manipulation. 
2. $x_{j}^{k}>x_{i}^{k}$. For this, we have three sub-cases.

(a) $j<i$. Then we have $x_{j}^{k}=x_{j}^{i-1}=\hat{x}_{j}^{i-1} \leq \hat{x}_{j}^{k}$, where the first transition follows due to Lemma 15, the second transition holds because manipulation by agent $i$ does not affect the allocations at step $i-1$, and the third transition follows since allocations are irrevocable.

(b) $j=i$. This cannot happen since we have assumed $x_{j}^{k}>x_{i}^{k}$ in this case.

(c) $j>i$. Since $x_{j}^{k}>x_{i}^{k}$, Lemma 15 implies that $x_{j}^{k}=x_{j}^{j}=E^{j}$, so $E^{j}>x_{i}^{k}$. Now using Lemma $16, E^{j}=x_{t}^{j-1} \cdot \min _{r \in R} d_{t r} / d_{j r}$ for some $t<i$. Then, $x_{t}^{j-1} \geq$ $x_{t}^{j-1} \cdot \min _{r \in R} d_{t r} / d_{j r}=E^{j}>x_{i}^{k} \geq x_{i}^{j-1}$, where the first transition follows since $\min _{r \in R} d_{t r} / d_{j r}$ is at most 1 and the last transition follows since allocations are irrevocable. Now Lemma 15 implies that $x_{t}^{j-1}=x_{t}^{i-1}$. Putting all the pieces together,

$$
\begin{aligned}
x_{j}^{k} & =E^{j}=x_{t}^{j-1} \cdot \min _{r \in R} d_{t r} / d_{j r}=x_{t}^{i-1} \cdot \min _{r \in R} d_{t r} / d_{j r}=x_{t}^{j-1} \cdot \min _{r \in R} d_{t r} / d_{j r} \\
& \leq \hat{x}_{t}^{j-1} \cdot \min _{r \in R} d_{t r} / d_{j r} \leq \hat{E}^{j} \leq \hat{x}_{j}^{j} \leq \hat{x}_{j}^{k},
\end{aligned}
$$

where the fifth transition follows since manipulation by agent $i$ does not change the allocation at step $i-1$, the sixth transition follows due to the definition of $\hat{E}^{j}$ (which is the value of $E^{j}$ after manipulation), the seventh transition follows due to the third constraint of the LP at step $j$ after manipulation, and the last transition follows since allocations are irrevocable.

Thus, we conclude that $\hat{x}_{j}^{k} \geq x_{j}^{k}$ for all agents $j \leq k$. $\square$ (Proof of Lemma 17)

Now, in the optimal solution of the LP at step $k$ without manipulation (i.e., in $\mathbf{A}^{k}$ ), the fourth constraint must be tight for some $t \leq k$ and $r \in R$ (otherwise $x_{j}^{k}$ for every $j \leq k$ and $M^{k}$ can be increased, contradicting the optimality of $M^{k}$ ). Thus,

$$
\sum_{j=1}^{k} x_{j}^{k} \cdot d_{j r}+(n-k) \cdot x_{t}^{k} \cdot d_{t r}=1 .
$$

Now consider the fourth constraint of the LP at step $k$ after manipulation for the same values of $t$ and $r$. For simplicity of notation, let $d_{j r}^{\prime}=d_{j r}$ for $j \neq i$. Then,

$$
\sum_{j=1}^{k} \hat{x}_{j}^{k} \cdot d_{j r}^{\prime}+(n-k) \cdot \hat{x}_{t}^{k} \cdot d_{t r}^{\prime}>\sum_{j=1}^{k} x_{j}^{k} \cdot d_{j r}+(n-k) \cdot x_{t}^{k} \cdot d_{t r}=1
$$

To justify the inequality, note that $\hat{x}_{i}^{k} \cdot d_{i r}^{\prime}>x_{i}^{k} \cdot d_{i r}$ by Equation (1) (as agent $i$ is strictly better off), and for any $j \leq k$ such that $j \neq i, \hat{x}_{j}^{k} \cdot d_{j r}^{\prime}=\hat{x}_{j}^{k} \cdot d_{j r} \geq x_{j}^{k} \cdot d_{j r}$ by Lemma 17. However, this shows that the allocation at step $k$ with manipulation violates the fourth constraint of the LP, which is impossible. Hence, a successful manipulation is impossible, that is, CAUTIOUS LP is SP.

Finally, note that at every step the LP has $O(n)$ variables and $O(n \cdot m)$ constraints, and there are $n$ such steps. Hence, the mechanism can be implemented in polynomial time. $\square$ (Proof of Theorem 11) 


\section{Experimental Results}

We presented two potentially useful mechanisms, DYNAMIC DRF and CAUTIOUS LP, each with its own theoretical guarantees. Our next goal is to analyze the performance of both mechanisms on real data, for two natural objectives: the sum of dominant shares (the maxsum objective) and the minimum dominant share (the maxmin objective) of the agents present in the system. ${ }^{1}$

We compare the objective function values achieved by the two mechanisms with certain lower and upper bounds. Since both mechanisms satisfy SI, their maxsum and maxmin objective values are provably lower bounded by $k / n$ and $1 / n$, respectively, at step $k$.

For upper bounds, we consider the omniscient (hence unrealistic) mechanisms that maximize the objectives in an offline setting where the mechanisms have complete knowledge of future demands. These mechanisms need to guarantee an EF extension only on the real future demands rather than on all possible future demands. The comparison of CAUTIOUS LP with these offline mechanisms demonstrates the loss CAUTIOUS LP (an online mechanism) suffers due to the absence of information regarding the future demands, that is, due to its cautiousness. Because DYNAMIC DRF is not required to have an EF extension, the offline mechanisms are not theoretical upper bounds for DYNAMIC DRF, but our experiments show that they provide upper bounds in practice.

As our data we use traces of real workloads on a Google compute cell, from a 7 hour period in 2011 (Hellerstein, 2010). The workload consists of tasks, where each task ran on a single machine, and consumed memory and one or more cores; the demands fit our model with two resources. For various values of $n$, we sampled $n$ random positive demand vectors from the traces and analyzed the value of the two objective functions under DYNAMIC DRF and CAUTIOUS LP along with the corresponding lower and upper bounds. We averaged over 1000 such simulations to obtain data points.

Figures 4(a) and 4(b) show the maxsum values achieved by the different mechanisms, for 20 agents and 100 agents respectively. The performance of our two mechanisms is nearly identical.

Figures 4(c) and 4(d) show the maxmin values achieved for 20 agents and 100 agents, respectively. Observe that DYNAMIC DRF performs better than CAUTIOUS LP for lower values of $k$, but performs worse for higher values of $k$. Intuitively, DYNAMIC DRF allocates more resources in early stages to satisfy DPO while CAUTIOUS LP cautiously waits. This results in the superior performance of DYNAMIC DRF in initial steps but it has fewer resources available and thus lesser flexibility for optimization in later steps, resulting in inferior performance near the end. In contrast, CAutious LP is later able to make up for its loss in early steps. Encouragingly, by the last step CAUTIOUS LP achieves near optimal maxmin value. For the same reason, unlike DYNAMIC DRF the maxmin objective value for CAUTIOUS LP monotonically increases as $k$ increases in our experiments (although it is easy to show that this is not always the case).

\section{Discussion}

We have presented a new model for resource allocation with multiple resources in dynamic environments that, we believe, can spark the study of dynamic fair division more generally. The model is directly applicable to data centers, clusters, and cloud computing, where the allocation of multiple resources is a key issue, and it significantly extends the previously studied static models. That said,

1. Under a cardinal notion of utility where the dominant share of an agent is its utility, the sum of dominant shares is the utilitarian social welfare and the minimum dominant share is the egalitarian social welfare. 


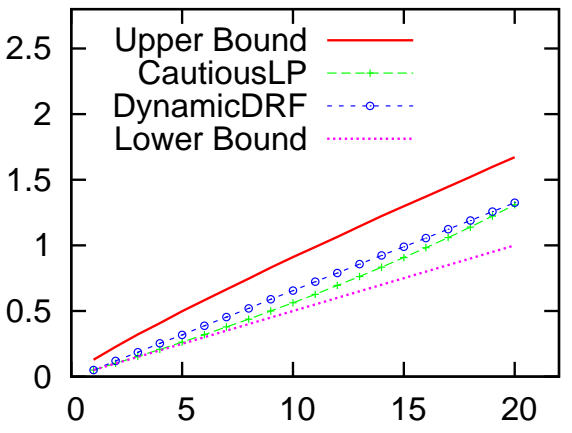

(a) Maxsum for 20 agents

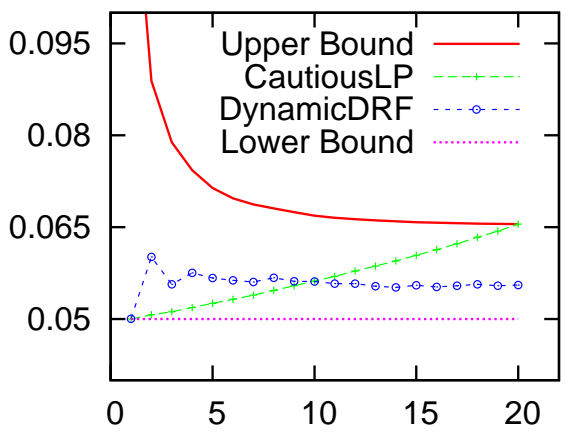

(c) Maxmin for 20 agents

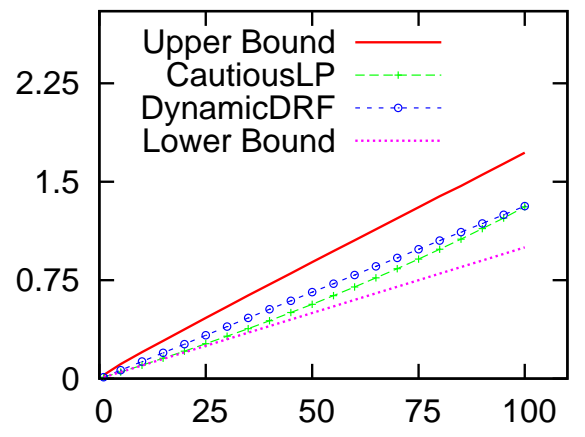

(b) Maxsum for 100 agents

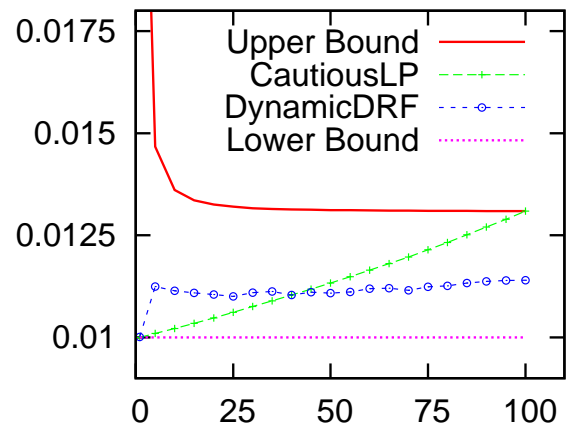

(d) Maxmin for 100 agents

Figure 4: The maxsum and maxmin objectives as a function of the time step $k$, for $n=20$ and $n=100$.

the model also gives rise to technical challenges that need to be tackled to capture more realistic settings.

First, our model assumes positive demands, that is, each agent requires every resource. To see how the positive demands assumption plays a role, recall that achieving EF and DPO is impossible. We established that dropping DPO leads to the trivial mechanism EQUAL SPLIT, which satisfies the remaining three properties; this is also true for possibly zero demands. When we dropped EF, we observed that the trivial mechanism DYNAMIC DICTATORSHIP satisfies SI, DPO and SP, and we subsequently suggested the improved mechanism DYNAMIC DRF that satisfies DEF in addition to SI, DPO and SP. Surprisingly though, it can be shown that neither DYNAMIC DiCTATORSHIP (see Example 2) nor DYNAMIC DRF are SP under possibly zero demands. ${ }^{2}$ In fact, despite significant effort, we were unable to settle the question of the existence of a mechanism that satisfies SI, DPO and SP under possibly zero demands.

Second, our analysis is restricted to the setting of divisible tasks, where agents value fractional quantities of their tasks. Parkes et. al. (2014) consider the indivisible tasks setting, where only

2. Under possibly zero demands, we modify DYNAMIC DICTATORSHIP and DYNAMIC DRF to continue allocating even when some resources become saturated so that they satisfy DPO. 
integral quantities of an agent's task are executed, albeit in a static environment. It can be shown that even forward EF - the weakest of all EF relaxations considered in this paper - is impossible to achieve along with DPO under indivisible tasks. It remains open to determine which relaxations of EF are feasible in dynamic resource allocation settings with indivisible tasks. While we restrict our attention to Leontief utilities, it should be noted that the desiderata we propose are well-defined in our dynamic setting with any utility function.

Third, while our model of fair division extends the classical model by introducing dynamics, and our results can directly inform the design of practical mechanisms, we do make the assumption that agents arrive over time but do not depart. In reality, agents may arrive and depart multiple times, and their preferences may also change over time (note that changing preferences can be modeled as a departure and simultaneous re-arrival with a different demand vector). Departures without re-arrivals are easy to handle; one can allocate the resources that become free in a similar way to allocations of entitlements, e.g., using DYNAMIC DRF (this scheme would clearly satisfy SI, DEF, and DPO, and it would be interesting to check whether it is also strategyproof). However, departures with re-arrivals immediately lead to daunting impossibilities. Note though that mechanisms that were designed for static settings performed well in realistic (fully dynamic) environments (Ghodsi et al., 2011), and it is quite likely that our mechanisms - which do provide theoretical guarantees for restricted dynamic settings - would yield even better performance in reality.

\section{Acknowledgements}

A preliminary version of this paper appeared in AAMAS'13. Procaccia and Shah were partially supported by the NSF under grant NSF CCF-1215883, and by a gift from the CMU-MSR Center for Computational Thinking.

\section{References}

Brams, S. J., \& Taylor, A. D. (1996). Fair Division: From Cake-Cutting to Dispute Resolution. Cambridge University Press.

Chen, Y., Lai, J. K., Parkes, D. C., \& Procaccia, A. D. (2010). Truth, justice, and cake cutting. In Proceedings of the 24th AAAI Conference on Artificial Intelligence (AAAI), pp. 756-761.

Chevaleyre, Y., Dunne, P. E., Endriss, U., Lang, J., Lemaître, M., Maudet, N., Padget, J., Phelps, S., Rodríguez-Aguilar, J. A., \& Sousa, P. (2006). Issues in multiagent resource allocation. Informatica, 30, 3-31.

Chevaleyre, Y., Endriss, U., Estivie, S., \& Maudet, N. (2007). Reaching envy-free states in distributed negotiation settings. In Proceedings of the 20th International Joint Conference on Artificial Intelligence (IJCAI), pp. 1239-1244.

Demers, A., Keshav, S., \& Shenker, S. (1989). Analysis and simulation of a fair queueing algorithm. In Proceedings of the ACM Symposium on Communications Architectures \& Protocols (SIGCOMM), pp. 1-12.

Dolev, D., Feitelson, D. G., Halpern, J. Y., Kupferman, R., \& Linial, N. (2012). No justified complaints: On fair sharing of multiple resources. In Proceedings of the 3rd Innovations in Theoretical Computer Science Conference (ITCS), pp. 68-75. 
Ghodsi, A., Sekar, V., Zaharia, M., \& Stoica, I. (2012). Multi-resource fair queueing for packet processing. In Proceedings of the ACM Symposium on Communications Architectures \& Protocols (SIGCOMM), pp. 1-12.

Ghodsi, A., Zaharia, M., Hindman, B., Konwinski, A., Shenker, S., \& Stoica, I. (2011). Dominant Resource Fairness: Fair allocation of multiple resource types. In Proceedings of the 8th USENIX Conference on Networked Systems Design and Implementation (NSDI), pp. 24-37.

Ghodsi, A., Zaharia, M., Shenker, S., \& Stoica, I. (2013). Choosy: Max-min fair sharing for datacenter jobs with constraints. In Proceedings of the 8th ACM European Conference on Computer Systems (EUROSYS), pp. 365-378.

Gutman, A., \& Nisan, N. (2012). Fair allocation without trade. In Proceedings of the 11th International Joint Conference on Autonomous Agents and Multi-Agent Systems (AAMAS), pp. 719-728.

Hellerstein, J. L. (2010). Google cluster data. Google research blog. Posted at http:// googleresearch.blogspot.com/2010/01/google-cluster-data.html.

Joe-Wong, C., Sen, S., Lan, T., \& Chiang, M. (2012). Multi-resource allocation: Fairness-efficiency tradeoffs in a unifying framework. In Proceedings of the 31st Annual IEEE International Conference on Computer Communications (INFOCOM), pp. 1206-1214.

Li, J., \& Xue, J. (2013). Egalitarian division under Leontief preferences. Economic Theory, 54(3), $597-622$.

Moulin, H. (2003). Fair Division and Collective Welfare. MIT Press.

Moulin, H., \& Stong, R. (2002). Fair queuing and other probabilistic allocation methods. Mathematics of Operations Research, 27(1), 1-30.

Parkes, D. C., Procaccia, A. D., \& Shah, N. (2014). Beyond Dominant Resource Fairness: Extensions, limitations, and indivisibilities. ACM Transactions on Economics and Computation. Forthcoming.

Procaccia, A. D. (2009). Thou shalt covet thy neighbor's cake. In Proceedings of the 21st International Joint Conference on Artificial Intelligence (IJCAI), pp. 239-244.

Walsh, T. (2011). Online cake cutting. In Proceedings of the 3rd International Conference on Algorithmic Decision Theory (ADT), pp. 292-305.

Zahedi, S. M., \& Lee, B. C. (2014). REF: Resource elasticity fairness with sharing incentives for multiprocessors. In Proceedings of the 19th International Conference on Architectural Support for Programming Languages and Operating Systems (ASPLOS), pp. 145-160. 